\title{
DISTRIBUTION FUNCTIONS AND THE RIEMANN ZETA FUNCTION*
}

\author{
BY \\ BØRGE JESSEN AND AUREL WINTNER
}

\section{INTRODUCTION}

The present paper starts with a systematic study of distribution functions in $k$-dimensional space and in particular of their infinite convolutions representing, in the language of the calculus of probability, the distributions arising by addition of an infinite number of independent random variables. The results are applied to almost periodic functions and in particular to the Riemann zeta function.

The proper method in dealing with distribution functions and their convolutions ("Faltungen") is the method of Fourier transforms, first applied systematically by Lévy in his book on the calculus of probability [40]. $\dagger$ The theorems concerning Fourier transforms which we need are collected at the beginning; for proofs we refer to papers of Bochner [2] and Haviland [28, 29]. These authors use Riemann-Radon integrals; we prefer for several reasons to work with Lebesgue-Radon integrals for which the proofs are simpler. Using these results on Fourier transforms we develop a general theory of infinite convolutions and in particular their convergence theory. This theory is completed at the end of the paper, where it is shown, by means of integrals in infinitely many dimensions, that the convergence problem of infinite convolutions is identical with the convergence problem of infinite series the terms of which are independent random variables, as considered by Khintchine and Kolmogoroff [37], Kolmogoroff [38], and Lévy [41]; incidentally we obtain a new treatment of the latter problem.

The dominating feature of the convolution process is its smoothing effect, although it is hardly possible to formulate a single theorem covering the whole situation. In the cases in which we are interested an appraisal of the Fourier transforms is the natural approach to the treatment of the question. This method has recently been applied in the case of circular equidistributions by Wintner [55]; in the present paper it will be applied to the more general case of distributions on convex curves, fundamental for the treatment of the zeta function. The results thus obtained are essentially finer than those obtained through geometrical considerations by Bohr and Jessen [19].

These results are then applied to the almost periodic functions of Bohr

* Presented to the Society, April 20, 1935 ; received by the editors July 9, 1934.

$\dagger$ Numbers in brackets refer to the Bibliography at the end of this paper. 
and their generalizations. Distribution problems for almost periodic functions have been considered from various points of view by several authors. Historically, we first mention the investigations of Bohr [4-14], Bohr and Courant [18], Bohr and Jessen [19, 20] and Jessen [31, 32] concerning Dirichlet series and in particular the Riemann zeta function, which are based on the theory of diophantine approximations (more particularly on the Kronecker-Weyl theorem). These investigations are methodically independent of the general theory of almost periodic functions which is, in the main, of a later date. Distribution functions for arbitrary real functions $x=x(t)$, almost periodic in the Bohr sense, were treated by Wintner [50-54] by transforming $t$-averages into $x$-averages and then applying the moment method of the calculus of probability. It was shown by Haviland [27] that the same method is valid also if $x(t)$ is complex-valued. In the special case of linearly independent exponents as considered by Wintner [53-55] it was essential to work not only with moments but also with Fourier transforms. It was shown by Bochner and Jessen [3] that the whole problem may be treated without applying the moment theory and considering only Fourier transforms, a method which holds in the case of generalized almost periodic functions also.

Applying this method in the present paper to the Riemann zeta function $\zeta(s)=\zeta(\sigma+i t)$ we obtain with regard to the functions $\log \zeta(s)$ and $\zeta(s)$ results which are in their kind essentially more precise than those obtained so far. The distribution function of $x(t)=\log \zeta(\sigma+i t)$ for a fixed $\sigma>\frac{1}{2}$ is obtained in the form of an infinite convolution. This expression for the distribution function of $\log \zeta(\sigma+i t)$ occurs in a geometrical form in the papers of Bohr and Jessen $[19,20]$, who have proved by elementary considerations that it possesses a continuous density. In the present paper it will be shown without recourse to this result that there exists a density possessing continuous partial derivatives of arbitrarily high order. In the case $\frac{1}{2}<\sigma<1$ it will even be shown that the density is a regular analytic function of the coordinates (which cannot be the case for $\sigma>1$ ). The distribution function of $x(t)=\zeta(\sigma+i t)$ itself is obtained from that of $x(t)=\log \zeta(\sigma+i t)$ by an exponential mapping. The method would enable us to discuss the dependence of the densities upon $\sigma$ also. In this direction we prove that the density of the distribution function of $x(t)=\log \zeta(\sigma+i t)$ tends uniformly to zero as $\sigma \rightarrow \frac{1}{2}$ and we also prove an analogous result with regard to the density of the distribution function of $x(t)=\zeta(\sigma+i t)$. It may be mentioned that the presentation is independent of the theory of diophantine approximations, which is replaced by a direct argument (using, of course, the same ideas as those in the proof of the Kronecker-Weyl theorem [48]).

In order to make the present paper independent of the papers mentioned 
above we have included, often with simplified proofs, some of the results of these papers. There is one aspect of the distribution problem regarding almost periodic functions and the zeta function not considered in the present paper, namely the problem of the $a$-points, studied by Bohr, Bohr and Jessen, Favard, and Jessen. We mention only that the methods of the present paper admit of applications to this problem also. The Bibliography at the end of the paper is intended to be complete as far as distribution problems for almost periodic functions are concerned.

\section{Distribution functions}

Let $R_{x}$ be a $k$-dimensional euclidean space with $x=\left(\xi_{1}, \cdots, \xi_{k}\right)$ as variable point. A completely additive, non-negative set function $\phi(E)$ defined for all Borel sets $E$ in $R_{x}$ and having the value 1 for $E=R_{x}$ will be called a distribution function in $R_{x}$. The notation for an integral with respect to $\phi$ will be

$$
\int_{E} f(x) \phi\left(d R_{x}\right)
$$

All integrals are understood in the Lebesgue-Radon sense (Radon [45], pp. 1324-1329). Our notation for ordinary Lebesgue integrals will be

$$
\int_{E} f(x) m\left(d R_{x}\right) \text {. }
$$

A set $E$ is called a continuity set of $\phi$ if $\phi\left(E^{\prime}\right)=\phi\left(E^{\prime \prime}\right)$ where $E^{\prime}$ denotes the set formed by all interior points of $E$ and $E^{\prime \prime}$ is the closure of $E$. There exists an at most enumerable set of real numbers such that at least those intervals $\alpha_{j}<\xi_{i}<\beta_{i}(j=1, \cdots, k)$ for which the numbers $\alpha_{j}, \beta_{i}$ do not belong to this set are continuity sets of $\phi$.

A sequence of distribution functions $\phi_{n}$ is said to be convergent if there exists a distribution function $\phi$ such that $\phi_{n}(E) \rightarrow \phi(E)$ for all continuity sets $E$ of the limit function $\phi$, which is then unique. The symbol $\phi_{n} \rightarrow \phi$ will be used only in this sense.

We have $\phi_{n} \rightarrow \phi$ if and only if the relation

$$
\int_{R_{x}} f(x) \phi_{n}\left(d R_{x}\right) \rightarrow \int_{R_{x}} f(x) \phi\left(d R_{x}\right)
$$

holds for all bounded continuous functions $f(x)$ in $R_{x}$. Furthermore, if $\phi_{n} \rightarrow \phi$ then

$$
\int_{R_{x}} f(x) \phi\left(d R_{x}\right) \leqq \liminf \int_{R_{x}} f(x) \phi_{n}\left(d R_{x}\right)
$$

holds for every non-negative, continuous function $f(x)$ in $R_{x}$. 
If $\phi_{1}, \phi_{2}$ is a pair of distribution functions, a new distribution function $\phi$ is defined by

$$
\phi(E)=\int_{R_{x}} \phi_{1}(E-x) \phi_{2}\left(d R_{x}\right) .
$$

Here $E-x$ denotes the set obtained from $E$ by the translation $-x$; the function $\phi_{1}(E-x)$ is a bounded Baire function so that the integral exists. ${ }^{*} \mathrm{We}$ call $\phi$ the convolution (Faltung) of $\phi_{1}$ and $\phi_{2}$ and denote it by $\phi=\phi_{1} * \phi_{2}$. It may be proved directly that $\phi_{1} * \phi_{2}=\phi_{2} * \phi_{1}$ and $\phi_{1} *\left(\phi_{2} * \phi_{3}\right)=\left(\phi_{1} * \phi_{2}\right) * \phi_{3}$, relations which also follow from the connection with the Fourier transforms discussed in \$3. For later application we notice that if $\psi_{n}=\phi_{1} * \cdots * \phi_{n}$ where $\phi_{1}, \cdots, \phi_{n}$ are distribution functions in $R_{x}$, and if $h(x)$ is a non-negative Baire function in $R_{x}$ then $\dagger$

$$
\int_{R_{x}} h(x) \psi_{n}\left(d R_{x}\right)=\int_{R_{x_{n}}} \phi_{n}\left(d R_{x_{n}}\right) \cdots \int_{R_{x_{1}}} h\left(x_{1}+\cdots+x_{n}\right) \phi_{1}\left(d R_{x_{1}}\right) .
$$

By the spectrum $\ddagger S=S(\phi)$ of a distribution function $\phi$ we understand the set of those points $x$ of $R_{x}$ for which $\phi(E)>0$ holds for any set $E$ containing $x$ as an interior point. $S$ is always a closed set containing at least one point. The point spectrum $P=P(\phi)$ is defined as the set of those points $x$ for which $\phi(x)>0$, where $x$ is to be understood as the Borel set consisting of the point $x$ alone. $P$ is at most enumerable and may be empty.

The vectorial sum $A+B$ of two point sets $A$ and $B$ in $R_{x}$ is defined as the set of those points in $R_{x}$ which may be represented in at least one way as a vector sum $a+b$ where $a$ and $b$ are points of $A$ and $B$ respectively. We agree to let $A+B$ denote the empty set if at least one of the sets $A$ and $B$ is empty. If $A$ and $B$ are both closed and bounded then so is $A+B$. We have $A+B$ $=B+A$ and $A+(B+C)=(A+B)+C$.

* That $g(x)=\phi_{1}(E-x)$ is a Baire function for any Borel set $E$ follows thus: The system of sets $E$ for which $g(x)$ is a Baire function is a Borel field; hence it is sufficient to consider the case where $E$ is an interval $\alpha_{j}<\xi_{j}<\beta_{j}(j=1, \cdots, k)$. Let now $h_{1}(x), h_{2}(x), \cdots$ be a sequence of continuous functions such that $h_{n}(x)=0$ when $x$ does not belong to $E$ and $h_{n}(x) \rightarrow 1$ when $x$ belongs to $E$, finally $0 \leqq h_{n}(x) \leqq 1$. Then

$$
g_{n}(x)=\int_{R_{u}} h_{n}(u+x) \phi_{1}\left(d R_{u}\right) \rightarrow \phi_{1}(E-x)=g(x)
$$

for all $x$. Since every $g_{n}(x)$ is continuous it follows that $g(x)$ is a Baire function.

$\dagger$ It is sufficient to verify (2.2) for $n=2$. In this case (2.2) is trivial if $h(x)$ is $=1$ in a Borel set $E$ and $=0$ elsewhere. Hence (2.2) holds for any $h(x)$ taking on but an enumerable number of values which implies that (2.2) holds for any Baire function $h(x) \geqq 0$. The relation (2.2) is understood in the sense that the finiteness of either side implies that of the other.

$\ddagger$ The above terminology, proposed by Wintner [57], is in accordance with that of Wirtinger and Hilbert and is therefore not identical with that of Wiener. 
Using this notation, we have for the spectrum and the point spectrum of a convolution the addition rules $S\left(\phi_{1} * \phi_{2}\right)=S\left(\phi_{1}\right)+S\left(\phi_{2}\right)$ and $P\left(\phi_{1} * \phi_{2}\right)$ $=P\left(\phi_{1}\right)+P\left(\phi_{2}\right)$.

By the limit $\lim B_{n}$ of a sequence of point sets $B_{n}$ in $R_{x}$ we understand the set of those points in $R_{x}$ which may be represented in at least one way as the limit of a sequence of points $x_{n}$, where $x_{n}$ belongs to $B_{n}$. This limit (which may be empty) always exists and is a closed set. By the vectorial sum $A_{1}+A_{2}+\cdots$ of an infinite sequence of sets $A_{1}, A_{2}, \cdots$ (in this order) we mean the set $\lim \left(A_{1}+\cdots+A_{n}\right)$.

If $\phi_{n} \rightarrow \phi$ the spectrum $S(\phi)$ is contained in $\lim S\left(\phi_{n}\right)$ but the two sets need not coincide. Between $P(\phi)$ and $\lim P\left(\phi_{n}\right)$ there is no connection.

A distribution function $\phi$ will be called continuous or discontinuous according as $P(\phi)$ is or is not empty. $\dagger$ We shall say that $\phi$ is purely discontinuous if $\phi(P(\phi))=1$. A distribution function $\phi$ will be called singular if it is continuous and there exists a Borel set $E$ of measure zero for which $\phi(E)=1$. Finally, a distribution function $\phi$ is called absolutely continuous if $\phi(E)=0$ for every Borel set $E$ of measure zero; this is the case if and only if there exists in $R_{x}$ a Lebesgue integrable point function $D(x)$ such that

$$
\phi(E)=\int_{E} D(x) m\left(d R_{x}\right)
$$

for any Borel set $E$; we call $D(x)$ the density of $\phi$.

Any distribution function $\phi$ may be written (Radon [45], pp. 1321-1322) in the form $\phi(E)=\alpha_{1} \phi_{1}(E)+\alpha_{2} \phi_{2}(E)+\alpha_{3} \phi_{3}(E)$ where $\alpha_{1}, \alpha_{2}, \alpha_{3}$ are nonnegative numbers having the sum 1 and $\phi_{1}, \phi_{2}, \phi_{3}$ are distribution functions such that $\phi_{1}$ is purely discontinuous, $\phi_{2}$ is singular, and $\phi_{3}$ is absolutely continuous; the three components $\alpha_{1} \phi_{1}, \alpha_{2} \phi_{2}, \alpha_{3} \phi_{3}$ are uniquely determined by $\phi$.

\section{FoURIER TRANSFORMS}

Let $R_{y}$ be the $k$-dimensional space with $y=\left(\eta_{1}, \cdots, \eta_{k}\right)$ as variable point and let $x y$ denote the scalar product of the vectors $x=\left(\xi_{1}, \cdots, \xi_{k}\right)$ and $y=\left(\eta_{1}, \cdots, \eta_{k}\right)$. If $\phi$ is a distribution function in $R_{x}$ then the integral

$$
\Lambda(y ; \phi)=\int_{R_{x}} e^{i x y} \phi\left(d R_{x}\right)
$$

defines in $R_{y}$ a function $\Lambda(y ; \phi)$ which is uniformly continuous and bounded, the maximum of its absolute value being $\Lambda(0 ; \phi)=1$. We call $\Lambda(y ; \phi)$ the Fourier transform of $\phi$. If $\Lambda(y ; \phi) \equiv \Lambda(y ; \psi)$ then $\phi=\psi$, that is, the correspond-

$\dagger$ We notice that there exist continuous distribution functions $\phi$ such that not every interval $\alpha_{j}<\xi_{j}<\beta_{j}(j=1, \cdots, k)$ is a continuity set of $\phi$ in the sense defined above. 
ence between the class of all distribution functions and the class of their Fourier transforms is a one-one correspondence.

If $\phi_{n} \rightarrow \phi$ then $\Lambda\left(y ; \phi_{n}\right) \rightarrow \Lambda(y ; \phi)$ holds uniformly in every sphere $|y| \leqq a$; conversely, if a sequence of Fourier transforms $\Lambda\left(y ; \phi_{n}\right)$ is uniformly convergent in every sphere $|y| \leqq a$ then the limit function also is the Fourier transform $\Lambda(y ; \phi)$ of a distribution function $\phi$ and $\phi_{n} \rightarrow \phi$. We may formulate this fact by saying that the one-one correspondence between the class of all distribution functions and their Fourier transforms is a continuous correspondence.

Finally we have for the Fourier transform of a convolution the multiplication rule $\Lambda\left(y ; \phi_{1} * \phi_{2}\right)=\Lambda\left(y ; \phi_{1}\right) \Lambda\left(y ; \phi_{2}\right)$. This, together with the uniqueness of the correspondence, implies immediately the relations $\phi_{1} * \phi_{2}=\phi_{2} * \phi_{1}$ and $\phi_{1} *\left(\phi_{2} * \phi_{3}\right)=\left(\phi_{1} * \phi_{2}\right) * \phi_{3}$ mentioned in $\$ 2$.

Let $\chi_{c}$ denote the distribution function whose spectrum consists of the single point $c$ in $R_{x}$ so that $\chi_{c}(E)=1$ or 0 according as $c$ is or is not contained in $E$; we have $\Lambda\left(y ; \chi_{c}\right)=e^{i c y}$ and in particular $\Lambda\left(y ; \chi_{0}\right) \equiv 1$. If $\psi$ is the distribution function $\psi(E)=\phi(E-c)$ then $\psi=\phi * \chi_{c}$; in particular, $\phi=\phi * \chi_{0}$ for every $\phi$. For later application we notice that if $\phi_{n} \rightarrow \phi$ and $\phi_{n} * \psi_{n} \rightarrow \phi$ then $\dagger$ $\psi_{n} \rightarrow \chi_{0}$.

If the integral

$$
\int_{R_{y}}|y|^{p \mid} \Lambda(y ; \phi) \mid m\left(d R_{y}\right)
$$

is finite for an integer $p \geqq 0$ then $\phi$ is absolutely continuous and its density $D(x)=D\left(\xi_{1}, \cdots, \xi_{k}\right)$, determined by the inversion formula

$$
D(x)=(2 \pi)^{-k} \int_{R_{y}} e^{-i x y} \Lambda(y ; \phi) m\left(d R_{y}\right),
$$

is continuous, approaches zero when $|x| \rightarrow \infty$, and possesses in the case $p>0$ continuous partial derivatives of order $\leqq p$ which may be obtained by differentiation under the integral sign and approach zero when $|x| \rightarrow \infty$. This is in particular the case if for some $\epsilon>0$

$$
\Lambda(y ; \phi)=O\left(|y|^{-(k+p+\epsilon)}\right) \text { as }|y| \rightarrow \infty .
$$

$\dagger$ This is proved in the following way: We know that $\Lambda\left(y ; \phi_{n}\right) \rightarrow \Lambda(y ; \phi)$ and $\Lambda\left(y ; \phi_{n}\right) \Lambda\left(y ; \psi_{n}\right)$ $\rightarrow \Lambda(y ; \phi)$ hold uniformly in every sphere $|y| \leqq a$ and wish to prove that $\Lambda\left(y ; \psi_{n}\right) \rightarrow \Lambda\left(y ; \chi_{0}\right) \equiv 1$ holds uniformly in every sphere $|y| \leqq a$. This is obvious if $\Lambda(y ; \phi) \neq 0$ for all $y$. Otherwise, since $\Lambda(0 ; \phi)=1$, we know at least that $\Lambda\left(y ; \psi_{n}\right) \rightarrow 1$ holds uniformly in a sufficiently small sphere $|y| \leqq a$. Now it is clear from the definition of the Fourier transform that if for an arbitrary distribution function $\psi$ and for some $y$ the value $\Lambda(y ; \psi)$ is near to 1 then the value $\Lambda(2 y ; \psi)$ is also near to 1 ; for that $\Lambda(y ; \psi)$ is near to 1 means that $e^{i x y}$ is near to 1 in a set $E$ in $R_{x}$ for which $\psi(E)$ is near to 1 and in this set $e^{2 i x y}$ is then also near to 1 . Since $\Lambda\left(y ; \psi_{n}\right) \rightarrow 1$ holds uniformly in $|y| \leqq a$ it follows that it holds uniformly in $|y| \leqq 2 a$ and by repetition that it holds uniformly in any sphere $|y| \leqq 2^{m} a$ which proves the theorem. 
A necessary condition for the absolute continuity of $\phi$ is that $\Lambda(y ; \phi) \rightarrow 0$ as $|y| \rightarrow \infty$ (Riemann-Lebesgue lemma).

If the estimate

$$
\Lambda(y ; \phi)=O\left(e^{-\Lambda|y|}\right) \text { as }|y| \rightarrow \infty
$$

holds for some $A>0$, it follows from the inversion formula by calculating the partial derivatives of $D(x)=D\left(\xi_{1}, \cdots, \xi_{k}\right)$ at an arbitrary point $x^{0}=\left(\xi_{1}^{0}, \cdots, \xi_{k}^{0}\right)$ of $R_{x}$ that $D(x)$ may be developed according to the powers of $\xi_{1}-\xi_{1}^{0}, \cdots, \xi_{k}-\xi_{k}^{0}$ into a power series convergent if $\left|\xi_{1}-\xi_{1}^{0}\right|<A / k^{1 / 2}, \cdots$, $\left|\xi_{k}-\xi_{k}^{0}\right|<A / k^{1 / 2}$ so that $D(x)$ is regular analytic in the whole real space $R_{x}$. If in particular (3.2) holds for arbitrarily large $A$ then $D(x)$ is an entire function of the $k$ variables $\xi_{1}, \cdots, \xi_{k}$. Cf. Wintner [59].

If $\phi$ is such that for an integer $p>0$ the integral

$$
\mathrm{M}_{p}(\phi)=\int_{R_{x}}|x|^{p} \phi\left(d R_{x}\right)
$$

is finite, then $\Lambda(y ; \phi)=\Lambda\left(\eta_{1}, \cdots, \eta_{k} ; \phi\right)$ possesses in $R_{y}$ continuous partial derivatives of order $\leqq p$ which may be obtained from the formula defining $\Lambda(y ; \phi)$ by differentiation under the integral sign. These derivatives become at $y=0$ the moments

$$
\mu_{q_{1}, \cdots, q_{k}}(\phi)=\int_{R_{x}} \xi_{1}^{q_{1}} \cdots \xi_{k}^{q_{k}} \phi\left(d R_{x}\right)
$$

of $\phi$ of order $q=q_{1}+\cdots+q_{k} \leqq p$, multiplied by the factor $i q$. For $q=1$ and $q=2$ we shall use the shorter notations

$$
\mu_{h}(\phi)=\int_{R_{x}} \xi_{h} \phi\left(d R_{x}\right) \text { and } \mu_{h, j}(\phi)=\int_{R_{x}} \xi_{h} \xi_{j} \phi\left(d R_{x}\right) .
$$

The existence of $\mathbf{M}_{p}(\phi)$ for one value of $p$ implies of course its existence for all smaller values of $p$. If $\mathbf{M}_{p}\left(\phi_{1}\right)$ and $\mathbf{M}_{p}\left(\phi_{2}\right)$ are finite and $\phi=\phi_{1}{ }^{*} \phi_{2}$, then $\mathrm{M}_{p}(\phi)$ also is finite for the inequality $\left|x_{1}+x_{2}\right|^{p} \leqq 2^{p}\left(\left|x_{1}\right|^{p}+\left|x_{2}\right|^{p}\right)$ implies by (2.2)

$$
\begin{aligned}
\mathrm{M}_{p}(\phi)=\int_{R_{x}}|x|^{p} \phi\left(d R_{x}\right) & =\int_{R_{x_{2}}} \phi_{2}\left(d R_{x_{2}}\right) \int_{R_{x_{1}}}\left|x_{1}+x_{2}\right|^{p} \phi_{1}\left(d R_{x_{1}}\right) \\
& \leqq 2^{p}\left(\mathrm{M}_{p}\left(\phi_{1}\right)+\mathrm{M}_{p}\left(\phi_{2}\right)\right) .
\end{aligned}
$$

If $\mathrm{M}_{1}(\phi)$ is finite we denote by $c=c(\phi)$ the point in $R_{x}$ having the moments of the first order $\mu_{1}(\phi), \cdots, \mu_{k}(\phi)$ as its coordinates so that $c(\phi)$ is the center of gravity of the mass distribution determined by $\phi$. We denote by $\tilde{\phi}$ the distribution function $\tilde{\phi}(E)=\phi(E+c(\phi))$ so that $c(\tilde{\phi})=0$ and $\Lambda(y ; \phi)$ 
$=e^{i c(\phi)} \nu \Lambda(y ; \tilde{\phi}) ;$ the spectrum of $\tilde{\phi}$ is $S(\tilde{\phi})=S(\phi)-c(\phi)$. If $\mathrm{M}_{1}\left(\phi_{1}\right)$ and $\mathrm{M}_{1}\left(\phi_{2}\right)$ are finite and $\phi=\phi_{1} * \phi_{2}$, then $c(\phi)=c\left(\phi_{1}\right)+c\left(\phi_{2}\right)$ and $\tilde{\phi}=\tilde{\phi}_{1} * \tilde{\phi}_{2}$.

If $\mathrm{M}_{2}(\phi)$ is finite we have $\mathrm{M}_{2}(\phi)=\mu_{1,1}(\phi)+\cdots+\mu_{k, k}(\phi)$ and $\mathrm{M}_{2}(\phi)$ $=\mathbf{M}_{2}(\tilde{\phi})+|c(\phi)|^{2}$, hence $\mathbf{M}_{2}(\tilde{\phi}) \leqq \mathbf{M}_{2}(\phi)$. If $\mathbf{M}_{2}\left(\phi_{1}\right)$ and $\mathbf{M}_{2}\left(\phi_{2}\right)$ are finite and $\phi=\phi_{1} * \phi_{2}$ then $\mathrm{M}_{2}(\tilde{\phi})=\mathrm{M}_{2}\left(\tilde{\phi}_{1}\right)+\mathrm{M}_{2}\left(\tilde{\phi}_{2}\right)$.

A point set, a set function, or a point function in $R_{x}$ or $R_{y}$ is said to be of radial symmetry if it is invariant under all rotations about the origin. A distribution function $\phi$ is of radial symmetry if and only if $\Lambda(y ; \phi)$ is of radial symmetry. Furthermore, if $R_{\xi}$ denotes a line through $x=0$ and $\omega$ the distribution function in $R_{\xi}$ defined by $\omega(F)=\phi(E)$, where $E$ consists of those $x$ of $R_{x}$ the projection of which on $R_{\xi}$ belongs to the given Borel set $F$ in $R_{\xi}$, then $\omega$ is of radial symmetry and $\Lambda(y ; \phi)=\Lambda(\eta, \omega)$ where $\eta= \pm|y|$. If the distribution function $\phi$ of radial symmetry is absolutely continuous then its density $D(x)$ also is of radial symmetry so that $D(x)=\delta(r)$ where $r=|x|$. Furthermore,

$$
\Lambda(y ; \phi)=2 \pi(2 \pi /|y|)^{k / 2-1} \int_{0}^{\infty} \delta(r) r^{k / 2} J_{k / 2-1}(r|y|) d r
$$

(Cauchy-Poisson). As to the explicit connection between $\phi$ and $\omega$ in the case $k=2$, cf. Wintner [55].

\section{CONVERgenCE CRITERIA FOR INFINITE CONVOLUTIONS}

If $\phi_{1}, \phi_{2}, \cdots$ is a sequence of distribution functions we say that the infinite convolution $\phi_{1} * \phi_{2} * \cdots$ is convergent if on placing $\psi_{n}=\phi_{1} * \cdots * \phi_{n}$ there exists a distribution function $\psi$ such that $\psi_{n} \rightarrow \psi$ as $n \rightarrow \infty$; we write then $\psi=\phi_{1} * \phi_{2} * \cdots$. Necessary and sufficient for the existence of the infinite convolution is that the infinite product $\Lambda\left(y ; \phi_{1}\right) \Lambda\left(y ; \phi_{2}\right) \cdots$ converges $\dagger$ uniformly in every sphere $|y| \leqq a$; we have then $\Lambda\left(y ; \phi_{1} * \phi_{2} * \cdots\right)=\Lambda\left(y ; \phi_{1}\right)$ $\cdot \Lambda\left(y ; \phi_{2}\right) \cdots$. Using the distribution function $\chi_{0}$ defined in $\S 3$ we have

THEOREM 1. A necessary and sufficient condition for the convergence of the infinite convolution $\phi_{1} * \phi_{2} * \cdots$ is that $\rho_{n, p} \rightarrow \chi_{0}$ as $n \rightarrow \infty$ where $\rho_{n, p}$ $=\phi_{n+1} * \cdots * \phi_{n+p}$ and $p$ depends on $n$ in an arbitrary way.

On placing $\psi_{n}=\phi_{1} * \cdots * \phi_{n}$ we have $\psi_{n+p}=\psi_{n} * \rho_{n, p}$. Consequently if $\psi_{n} \rightarrow \psi$ we have also $\psi_{n} * \rho_{n, p} \rightarrow \psi$, hence $\rho_{n, p} \rightarrow \chi_{0}$ by a result of $\S 3$. Conversely, if $\rho_{n, p} \rightarrow \chi_{0}$ then $\Lambda\left(y ; \rho_{n, p}\right)=\Lambda\left(y ; \phi_{n+1}\right) \cdots \Lambda\left(y ; \phi_{n+p}\right) \rightarrow 1$ uniformly in every sphere $|y| \leqq a$, hence $\Lambda\left(y ; \phi_{1}\right) \Lambda\left(y ; \phi_{2}\right) \cdots$ converges uniformly in every sphere $|y| \leqq a$.

$\dagger$ Throughout the paper convergence of an infinite product is meant in the sense that the product of the $n$ first factors approaches a limit so that the vanishing of the limit is not excluded. 
THEOREM 2. If $\psi=\phi_{1} * \phi_{2} * \cdots$ is convergent, then so is $\rho_{n}=\phi_{n+1} * \phi_{n+2} * \cdots$ for every $n$ and $\rho_{n} \rightarrow \chi_{0}$ as $n \rightarrow \infty$.

The convergence of $\phi_{n+1} * \phi_{n+2} * \cdots$ follows from Theorem 1. Also, $\psi_{n} * \rho_{n}=\psi$ and $\psi_{n} \rightarrow \psi$, hence $\rho_{n} \rightarrow \chi_{0}$.

These theorems enable us to prove that the addition rule for spectra holds for infinite convolutions also:

THEOREM 3. If $\psi=\phi_{1} * \phi_{2} * \cdots$ is convergent then $S(\psi)=S\left(\phi_{1}\right)$ $+S\left(\phi_{2}\right)+\cdots$.

The theorem states that $S(\psi)=\lim S\left(\psi_{n}\right)$ where $\psi_{n}=\phi_{1} * \cdots * \phi_{n}$. We know from $\$ 2$ that $S(\psi)$ is contained in $\lim S\left(\psi_{n}\right)$ and shall prove that $\lim S\left(\psi_{n}\right)$ is contained in $S(\psi)$. Let $x_{0}$ be a point of $\lim S\left(\psi_{n}\right)$ and let $C_{\epsilon}$ denote the sphere $|x|<\epsilon$ where $\epsilon>0$ is arbitrary; then $\psi_{n}\left(x_{0}+C_{\epsilon}\right)>0$ for $n$ sufficiently large. Now $\psi=\psi_{n} * \rho_{n}$, hence

$$
\begin{aligned}
\psi\left(x_{0}+C_{2 \epsilon}\right) & =\int_{R_{x}} \psi_{n}\left(x_{0}+C_{2 \epsilon}-x\right) \rho_{n}\left(d R_{x}\right) \geqq \int_{C_{\epsilon}} \psi_{n}\left(x_{0}+C_{2 \epsilon}-x\right) \rho_{n}\left(d R_{x}\right) \\
& \geqq \psi_{n}\left(x_{0}+C_{\epsilon}\right) \int_{C_{\epsilon}} \rho_{n}\left(d R_{x}\right)=\psi_{n}\left(x_{0}+C_{\epsilon}\right) \rho_{n}\left(C_{\epsilon}\right) .
\end{aligned}
$$

Since $\rho_{n} \rightarrow \chi_{0}$ we have $\rho_{n}\left(C_{\epsilon}\right) \rightarrow 1$ as $n \rightarrow \infty$ for any fixed $\epsilon$. Hence $\psi\left(x_{0}+C_{2 \epsilon}\right)>0$ for any $\epsilon$ which means that $x_{0}$ belongs to $S(\psi)$.

The above proof is a generalization of a proof in Bohr and Jessen [19].

On imposing conditions on the distribution functions we obtain convergence criteria which will be used later on.

THEOREM 4. If $\mathrm{M}_{2}\left(\phi_{n}\right)$ is finite for every $n$ then the convergence of the two series

$$
c\left(\phi_{1}\right)+c\left(\phi_{2}\right)+\cdots \text { and } \mathrm{M}_{2}\left(\tilde{\phi}_{1}\right)+\mathrm{M}_{2}\left(\tilde{\phi}_{2}\right)+\cdots
$$

implies the convergence of $\psi=\phi_{1} * \phi_{2} * \ldots$. Furthermore, $\mathrm{M}_{2}(\psi)$ is finite; finally,

$$
c(\psi)=c\left(\phi_{1}\right)+c\left(\phi_{2}\right)+\cdots \text { and } \mathbf{M}_{2}(\tilde{\psi})=\mathbf{M}_{2}\left(\tilde{\phi_{1}}\right)+\mathbf{M}_{2}\left(\tilde{\phi_{2}}\right)+\cdots .
$$

Since $\mathrm{M}_{2}\left(\tilde{\phi}_{n}\right) \leqq \mathrm{M}_{2}\left(\phi_{n}\right)$ is finite, $\Lambda\left(y ; \tilde{\phi}_{n}\right)$ possesses continuous partial derivatives of order $\leqq 2$ and those of the first order vanish at $y=0$ in virtue of $c\left(\tilde{\phi}_{n}\right)=0$. Hence

$$
\Lambda\left(y ; \tilde{\phi}_{n}\right)=1+\sum_{h=1}^{k} \sum_{j=1}^{k}\left[\Re \Lambda_{\eta_{h}, \eta_{j}}\left(\theta^{\prime} y ; \tilde{\phi}_{n}\right)+i \Im \Lambda_{\eta_{h}, \eta_{j}}\left(\theta^{\prime \prime} y ; \tilde{\phi}_{n}\right)\right] \eta_{h} \eta_{j}
$$

where the subscripts denote partial differentiations and $0<\theta^{\prime}<1,0<\theta^{\prime \prime}<1$. 
Since every partial derivative of second order of $\Lambda\left(y ; \tilde{\phi}_{n}\right)$ has an absolute value $\leqq \mathrm{M}_{2}\left(\tilde{\phi}_{n}\right)$ we have

$$
\left|\Lambda\left(y ; \tilde{\phi}_{n}\right)-1\right| \leqq 2 k^{2} \mathrm{M}_{2}\left(\tilde{\phi}_{n}\right)|y|^{2}
$$

which proves the convergence of $\omega=\tilde{\phi}_{1} * \tilde{\phi}_{2 *} \cdots$. The relation $\mathrm{M}_{2}\left(\tilde{\phi}_{1} * \cdots * \tilde{\phi}_{n}\right)$ $=\mathrm{M}_{2}\left(\tilde{\phi}_{1}\right)+\cdots+\mathrm{M}_{2}\left(\tilde{\phi}_{n}\right)$ implies by (2.1) that $\mathrm{M}_{2}(\omega)$ is finite, namely $\leqq \mathrm{M}_{2}\left(\tilde{\phi}_{1}\right)+\mathrm{M}_{2}\left(\tilde{\phi}_{2}\right)+\cdots$. Now it follows from (4.1) that the derivatives of the first and second order of $\Lambda(y ; \omega)$ at $y=0$ are the limits of the corresponding derivativ s of $\Lambda\left(y ; \tilde{\phi}_{1} * \cdots * \tilde{\phi}_{n}\right)$ as $n \rightarrow \infty$, so that $c(\omega)=0$, i.e., $\omega=\tilde{\omega}$, and $\mathbf{M}_{2}(\omega)=\mathbf{M}_{2}\left(\tilde{\phi}_{1}\right)+\mathbf{M}_{2}\left(\tilde{\phi}_{2}\right)+\cdots$. Finally, the relation $\Lambda\left(y ; \phi_{n}\right)=e^{i c\left(\phi_{n}\right) v} \Lambda\left(y ; \tilde{\phi}_{n}\right)$ and the convergence of $c\left(\phi_{1}\right)+c\left(\phi_{2}\right)+\cdots$ implies the convergence of $\psi=\phi_{1} * \phi_{2} * \cdots$ and also gives $c(\psi)=c\left(\phi_{1}\right)+c\left(\phi_{2}\right)+\cdots$ and $\tilde{\psi}=\omega$.

Examples show that the converse of Theorem 4 is false. We have, however, the following theorem:

THEOREM 5. If all spectra $S\left(\phi_{n}\right)$ are contained in a fixed sphere $|x| \leqq K$ then the convergence of the two series

$$
c\left(\phi_{1}\right)+c\left(\phi_{2}\right)+\cdots \text { and } \mathbf{M}_{2}\left(\tilde{\phi}_{1}\right)+\mathbf{M}_{2}\left(\tilde{\phi}_{2}\right)+\cdots
$$

is necessary and sufficient for the convergence of $\psi=\phi_{1} * \phi_{2} * \ldots$.

The sufficiency is implied by Theorem 4 . In order to prove the necessity we first show that the convergence of $\psi=\phi_{1} * \phi_{2} * \cdots$ implies the convergence of $\mathrm{M}_{2}\left(\tilde{\phi}_{1}\right)+\mathrm{M}_{2}\left(\tilde{\phi}_{2}\right)+\cdots$. For suppose that $\mathrm{M}_{2}\left(\tilde{\phi}_{1}\right)+\mathrm{M}_{2}\left(\tilde{\phi}_{2}\right)+\cdots$ is divergent; then it follows from $\mathbf{M}_{2}\left(\tilde{\phi}_{n}\right)=\mu_{1,1}\left(\tilde{\phi}_{n}\right)+\cdots+\mu_{k, k}\left(\tilde{\phi}_{n}\right)$ that for some value of $j$ the series $\mu_{i, j}\left(\tilde{\phi}_{1}\right)+\mu_{j, j}\left(\tilde{\phi}_{2}\right)+\cdots$ is divergent. We choose $y=\left(0, \cdots, 0, \eta_{i}, 0, \cdots, 0\right)$ so that

$$
\Lambda\left(y ; \tilde{\phi}_{n}\right)=\int_{R_{x}} e^{i \xi i \eta j} \tilde{\phi}_{n}\left(d R_{x}\right) .
$$

Instead of integrating over $R_{x}$ it is sufficient to integrate over the sphere $|x| \leqq 2 K$ which contains $S\left(\tilde{\phi}_{n}\right)=S\left(\phi_{n}\right)-c\left(\phi_{n}\right)$. Choose now an $\epsilon>0$ so small that for $|t|<2 K \epsilon$

$$
0 \leqq \cos t \leqq 1-t^{2} / 4 \text { and } \sin t=t+h(t) \text { where }|h(t)| \leqq t^{2} / 8 .
$$

Then $\left|\xi_{j} \eta_{j}\right|<2 K \epsilon$ in $|x| \leqq 2 K$ if $\left|\eta_{j}\right|<\epsilon$, hence

$$
0 \leqq \Re \Lambda\left(y ; \tilde{\phi}_{n}\right) \leqq 1-\eta_{j}^{2} \mu_{j, j}\left(\tilde{\phi}_{n}\right) / 4 \text { and }\left|\Im \Lambda\left(y ; \tilde{\phi}_{n}\right)\right| \leqq \eta_{j}^{2} \mu_{j, i}\left(\tilde{\phi}_{n}\right) / 8
$$

so. that

$$
\left|\Lambda\left(y ; \phi_{n}\right)\right|=\left|\Lambda\left(y ; \tilde{\phi}_{n}\right)\right| \leqq 1-\eta_{j}^{2} \mu_{j, j}\left(\tilde{\phi}_{n}\right) / 8
$$


Hence the divergence of $\mu_{j, j}\left(\tilde{\phi}_{1}\right)+\mu_{j, j}\left(\tilde{\phi}_{2}\right)+\cdots$ implies that $\Lambda(y ; \psi)$ $=\Lambda\left(y ; \phi_{1}\right) \Lambda\left(y ; \phi_{2}\right) \cdots=0$ for points $y$ arbitrarily near to $y=0$ and thus leads to a contradiction. Consequently $\mathrm{M}_{2}\left(\tilde{\phi}_{1}\right)+\mathrm{M}_{2}\left(\tilde{\phi}_{2}\right)+\cdots$ is convergent. In order to prove that $c\left(\phi_{1}\right)+c\left(\phi_{2}\right)+\cdots$ also converges we observe that by the proof of Theorem 4 the convergence of $\mathbf{M}_{2}\left(\tilde{\phi}_{1}\right)+\mathbf{M}_{2}\left(\tilde{\phi}_{2}\right)+\cdots$ inplies the convergence of $\tilde{\phi}_{1} * \tilde{\phi}_{2} * \ldots$. Since $\Lambda\left(y ; \phi_{1} * \ldots * \phi_{n}\right)=e^{i c_{n} v} \Lambda\left(y ; \tilde{\phi}_{1} * \ldots * \tilde{\phi}_{n}\right)$ where $c_{n}=c\left(\phi_{1}\right)+\cdots+c\left(\phi_{n}\right)$, we conclude that $e^{i c_{n} y}$ tends in a sufficiently small sphere $|y| \leqq a$ to a limit as $n \rightarrow \infty$. This is only possible if $c\left(\phi_{1}\right)$ $+c\left(\phi_{2}\right)+\cdots$ converges.

The infinite convolution $\psi=\phi_{1} * \phi_{2} * \ldots$ will be called absolutely convergent if it is convergent in any order of the terms; the connection with the Fourier transforms shows that $\psi$ is then also independent of the order of the terms. From Theorems 4 and 5 follows immediately

THEOREM 6. If $\mathrm{M}_{2}\left(\phi_{n}\right)$ is finite for every $n$, then the convergence of the two series

$$
\left|c\left(\phi_{1}\right)\right|+\left|c\left(\phi_{2}\right)\right|+\cdots \text { and } \mathbf{M}_{2}\left(\tilde{\phi}_{1}\right)+\mathbf{M}_{2}\left(\tilde{\phi}_{2}\right)+\cdots
$$

implies the absolute convergence of $\phi_{1} * \phi_{2} * \ldots$. If all spectra $S\left(\phi_{n}\right)$ are contained in a fixed sphere $|x| \leqq K$ then the converse is also true.

We notice that, since $\mathrm{M}_{2}\left(\phi_{n}\right)=\mathrm{M}_{2}\left(\tilde{\phi}_{n}\right)+\left|c\left(\phi_{n}\right)\right|^{2}$, the convergence of the two series is equivalent to the convergence of the series

$$
\left|c\left(\phi_{1}\right)\right|+\left|c\left(\phi_{2}\right)\right|+\cdots \text { and } \mathbf{M}_{2}\left(\phi_{1}\right)+\mathbf{M}_{2}\left(\phi_{2}\right)+\cdots \cdot
$$

Further theorems on infinite convolutions will be given in $\$ 16$.

\section{CONVOLUTIONS OF SPHERICAL EQUIDISTRIBUTIONS}

Let the dimension number $k$ be $>1$ and let $S$ denote the sphere $|x|=r$ where $r>0$. As equidistribution on $S$ we denote the distribution function $\phi(E)$ which for a given Borel set $E$ is the $(k-1)$-dimensional measure of $E S$, divided by that of $S$ itself. We have $S(\phi)=S, c(\phi)=0$ and $\mathrm{M}_{2}(\phi)=r^{2}$. Let $S_{1}, S_{2}, \cdots$ denote the spheres $|x|=r_{1},|x|=r_{2}, \cdots$, and $\phi_{1}, \phi_{2}, \cdots$ the corresponding equidistributions. If $\phi_{1} * \phi_{2} * \cdots$ is convergent then $\phi_{n} \rightarrow \chi_{0}$ in virtue of Theorem 1 , hence $r_{n} \rightarrow 0$. From Theorems 5 and 6 follows therefore

THEOREM 7. The convergence of the series $r_{1}{ }^{2}+r_{2}{ }^{2}+\cdots$ is necessary and sufficient both for the convergence and for the absolute convergence of the infinite convolution $\phi_{1} * \phi_{2} * \cdots$.

The Fourier transform $\Lambda(y ; \phi)$ of the spherical equidistribution $\phi$ is the mean value of $e^{i x y}$ on $S$. If $y$ is fixed and $x$ varies on $S$ we may write $x y=r|y| \cos \theta, 0 \leqq \theta \leqq \pi ;$ then $\phi(d S)=A_{k} \sin ^{k-2} \theta d \theta$, where $d S$ is the portion 
of $S$ corresponding to the interval $(\theta, \theta+d \theta)$ and $A_{k}=\pi^{-1 / 2} \Gamma\left(\frac{1}{2} k\right) / \Gamma\left(\frac{1}{2} k-\frac{1}{2}\right)$. Hence

$$
\Lambda(y ; \phi)=A_{k} \int_{0}^{\pi} e^{i r|y| \cos \theta} \sin ^{k-2} \theta d \theta=B_{k} J_{k / 2-1}(r|y|) /(r|y|)^{k / 2-1}
$$

where $B_{k}=2^{k / 2-1} \Gamma\left(\frac{1}{2} k\right)$. Since $J_{\nu}(u)=O\left(u^{-1 / 2}\right)$ as $u \rightarrow \infty$ we have

$$
\Lambda(y ; \dot{\phi})=O\left(|y|^{1 / 2-k / 2}\right) \text { as }|y| \rightarrow \infty \text {. }
$$

Let now $\phi_{1}, \phi_{2}, \cdots$ be the spherical equidistributions considered above. It follows from (5.1) that $\Lambda\left(y ; \phi_{1} * \cdots * \phi_{n}\right)$ satisfies (3.1) if $n>2(k+p)$ $/(k-1)$ and that in the case of convergence $\Lambda\left(y ; \phi_{1} * \phi_{2} * \cdots\right)$ satisfies (3.1) for every $p$ in virtue of $\left|\Lambda\left(y ; \phi_{n}\right)\right| \leqq 1$. Hence we have

THEOREM 8. The convolution $\psi_{n}=\phi_{1} * \cdots * \phi_{n}$ of $n$ spherical equidistributions is absolutely continuous with a continuous density $D_{n}(x)$ whenever $n>2 k /(k-1)$, and $D_{n}(x)=D_{n}\left(\xi_{1}, \cdots, \xi_{k}\right)$ possesses continuous partial derivatives of order $\leqq p$ whenever $n>2(k+p) /(k-1)$. If $\psi=\phi_{1} * \phi_{2} * \cdots$ is convergent, it is absolutely continuous and its density $D(x)=D\left(\xi_{1}, \cdots, \xi_{k}\right)$ is continuous and possesses continuous partial derivatives of all orders.

The above argument is that applied by Wintner [55] in the case $k=2$.

Remark. In view of the fact that the functions $\Lambda\left(y ; \phi_{1} * \ldots * \phi_{n}\right)$ are estimated by (3.1) uniformly for all $n>2(k+p) /(k-1)$, it follows from the uniform convergence of $\Lambda\left(y ; \phi_{1} * \ldots * \phi_{n}\right)$ to $\Lambda\left(y ; \phi_{1} * \phi_{2} * \ldots\right)$ in any sphere $|y| \leqq a$ in the case where $\phi_{1} * \phi_{2} * \cdots$ converges that

$$
\int_{R_{y}}|y|^{p}\left|\Lambda\left(y ; \phi_{1} * \cdots * \phi_{n}\right)-\Lambda\left(y ; \phi_{1} * \phi_{2} * \cdots\right)\right| m\left(d R_{y}\right) \rightarrow 0 \text { as } n \rightarrow \infty \text {. }
$$

This implies that $D_{n}(x)$ and its partial derivatives converge uniformly to $D(x)$ and its partial derivatives when $n \rightarrow \infty$. In the case where the series $r_{1}^{2}+r_{2}^{2}+\cdots$ is divergent it may easily be shown that $\Lambda\left(y ; \phi_{1} * \ldots * \phi_{n}\right) \rightarrow 0$ uniformly for $|y| \geqq \epsilon$ where $\epsilon>0$ is arbitrarily small. This implies that

$$
\int_{R_{y}}|y|^{p}\left|\Lambda\left(y ; \phi_{1} * \cdots * \phi_{n}\right)\right| m\left(d R_{y}\right) \rightarrow 0 \text { as } n \rightarrow \infty,
$$

so that in this case $D_{n}(x)$ and its partial derivatives tend uniformly to zero when $n \rightarrow \infty$. This is a more precise statement than merely saying that the infinite convolution $\phi_{1} * \phi_{2} * \cdots$ does not converge.

Since all $\phi_{n}$ are of radial symmetry, the same property holds for their convolutions and hence for the densities $D_{n}(x)$ and $D(x)$. For the spectra $S\left(\phi_{1} * \cdots * \phi_{n}\right)=S_{1}+\cdots+S_{n}$ and $S\left(\phi_{1} * \phi_{2} * \cdots\right)=S_{1}+S_{2}+\cdots$ one easily finds (cf. Bohr [6]) 
THEOREM 9. $S\left(\phi_{1} * \cdots * \phi_{n}\right)$ is the set $a_{n} \leqq|x| \leqq b_{n}$ where $b_{n}=r_{1}+\cdots+r_{n}$ and $a_{n}=0$ unless one of the $r$ 's in $r_{1}+\cdots+r_{n}$, say $r_{1}$, is larger than the sum of the other $r$ 's, in which case $a_{n}=r_{1}-\left(r_{2}+\cdots+r_{n}\right)$. If $r_{1}+r_{2}+\cdots$ is convergent then $S\left(\phi_{1} * \phi_{2} * \cdots\right)$ is the set $a \leqq|x| \leqq b$ where $b=r_{1}+r_{2}+\cdots$ and $a=0$ unless one of the r's, say $r_{1}$, is larger than the sum of all other $r$ 's, in which case $a=r_{1}-\left(r_{2}+r_{3}+\cdots\right)$. If $r_{1}+r_{2}+\cdots$ is divergent $\left(\right.$ but $r_{1}{ }^{2}+r_{2}{ }^{2}+\cdots$ is convergent) then $S\left(\phi_{1} * \phi_{2} * \cdots\right)$ is the whole $R_{x}$.

Remark. It is not difficult to determine the points $x$ for which $D_{n}(x)$ or $D(x)$ is positive. We prove in this direction only that in the case where $r_{1}+r_{2}+\cdots$ is divergent $D(x)$ is positive for all $x$ (from $S\left(\phi_{1} * \phi_{2} * \cdots\right)=R_{x}$ follows only that $D(x)$ is not identically zero in any sphere). On denoting by $D^{\prime}(x)$ and $D^{\prime \prime}(x)$ the densities of $\phi_{1} * \phi_{3} * \cdots$ and $\phi_{2} * \phi_{4} * \cdots$ we have

$$
D(x)=\int_{R_{u}} D^{\prime}(x-u) D^{\prime \prime}(u) m\left(d R_{u}\right)
$$

At least one of the two functions $D^{\prime}(x)$ and $D^{\prime \prime}(x)$ is not identically zero in any sphere; since the other function is positive in some sphere, we have $D(x)>0$ for every $x$.

For later reference we collect the main results for the case $k=2$ as a particular theorem.

THEOREM 10. If $\phi_{n}$ is the circular equidistribution on $|x|=r_{n}$ then $\psi_{n}=\phi_{1} * \cdots * \phi_{n}$ is absolutely continuous with a continuous density $D_{n}(x)$ if $n>4$ and $D_{n}(x)=D_{n}\left(\xi_{1}, \xi_{2}\right)$ possesses continuous partial derivatives of order $\leqq p$ if $n>4+2 p$. The convergence of $r_{1}{ }^{2}+r_{2}{ }^{2}+\cdots$ is necessary and sufficient both for the convergence and for the absolute convergence of the infinite convolution $\phi_{1} * \phi_{2} * \cdots$ and $\psi=\phi_{1} * \phi_{2} * \cdots$ is then absolutely continuous and its density $D(x)=D\left(\xi_{1}, \xi_{2}\right)$ is continuous and possesses continuous partial derivatives of arbitrarily high order. Finally, $D(x)>0$ for all $x$ if $r_{1}+r_{2}+\cdots$ diverges.

Finer results regarding $D(x)$ are contained in Theorems 15 and 16.

The results of this section could also be stated in terms of the so-called random walk problem; cf. Lord Rayleigh [46], Lüneburg [42].

\section{INFINITE CONVOLUTIONS OF SYMMETRIC BERNOULLI DISTRIBUTIONS}

Let the dimension number $k$ be $=1$. Let $S$ denote the set consisting of the two points $x= \pm r$ where $r>0$ and let $\phi(E)$ be the distribution function which is $0, \frac{1}{2}$ or 1 according as $E$ contains neither, one or both of these points so that $\Lambda(y ; \phi)=\frac{1}{2}\left(e^{-i r y}+e^{i r y}\right)=\cos r y$ and $S(\phi)=P(\phi)=S$. This $\phi$ is the analogue for $k=1$ of the spherical equidistribution. We have $c(\phi)=0$ and $\mathrm{M}_{2}(\phi)=r^{2}$. 
Hence if $S_{1}, S_{2}, \cdots$ denote the sets $x= \pm r_{1}, x= \pm r_{2}, \cdots$ and $\phi_{1}, \phi_{2}, \cdots$ the corresponding distribution functions, Theorem 7 holds again. On placing $\psi_{n}=\phi_{1} * \cdots * \phi_{n}$ we have $\psi_{n}(E)=h / 2^{n}$ if $E$ contains $h$ of the $2^{n}$ points $\pm r_{1} \pm \cdots \pm r_{n}$ which form the spectrum $S\left(\psi_{n}\right)=P\left(\psi_{n}\right)=S_{1}+\cdots+S_{n}$. It follows therefore from Theorem 3 that if $r_{1}^{2}+r_{2}^{2}+\cdots$ is convergent, so that $\psi=\phi_{1} * \phi_{2} * \cdots$ exists, then $S(\psi)$ is either a bounded set or the whole $R_{x}$ according as $r_{1}+r_{2}+\cdots$ is convergent or divergent. Furthermore, $P(\psi)$ is always empty. For suppose that there exists a point $x$ so that $\psi(x)>0$. Let us write $\psi=\sigma_{n} * \phi_{n}$ where $\sigma_{n}=\phi_{1} * \cdots * \phi_{n-1} * \phi_{n+1} * \cdots ;$; then $\psi(x)$ $=\frac{1}{2}\left(\sigma_{n}\left(x-r_{n}\right)+\sigma_{n}\left(x+r_{n}\right)\right)$ and similarly $\psi\left(x-2 r_{n}\right)=\frac{1}{2}\left(\sigma_{n}\left(x-3 r_{n}\right)+\sigma_{n}\left(x-r_{n}\right)\right)$, $\psi\left(x+2 r_{n}\right)=\frac{1}{2}\left(\sigma_{n}\left(x+r_{n}\right)+\sigma_{n}\left(x+3 r_{n}\right)\right)$ so that $\psi\left(x-2 r_{n}\right)+\psi\left(x+2 r_{n}\right) \geqq \psi(x)$. We choose a positive integer $p$ such that $\psi(x)>1 / p$ and determine then $p$ numbers $n_{1}, \cdots, n_{p}$ such that $r_{n_{1}}>\cdots>r_{n_{p}}$ which is possible in virtue of $r_{n} \rightarrow 0$. Then the numbers $x \pm r_{n_{1}}, \cdots, x \pm r_{n_{p}}$ are all distinct and the sum of the corresponding values $\psi\left(x-2 r_{n_{1}}\right)+\psi\left(x+2 r_{n_{1}}\right), \cdots, \psi\left(x-2 r_{n_{p}}\right)+\psi\left(x+2 r_{n_{p}}\right)$ is $\geqq p \psi(x)>1$ which is impossible. This proves that $P(\psi)$ is empty, i.e., that $\psi$ is continuous. From Theorem 35, to be proved later, we conclude therefore that $\psi$ is either singular or absolutely continuous. On collecting our results we have

THEOREM 11. The infinite convolution $\phi_{1} * \phi_{2} * \cdots$, where $\phi_{n}(E)=0, \frac{1}{2}$, or 1 according as $E$ contains neither, one, or both of the points $x= \pm r_{n}$, is convergent, and then also absolutely convergent, if and only if the series $r_{1}^{2}+r_{2}^{2}+\cdots$ is convergent. The Fourier transform of $\psi=\phi_{1} * \phi_{2} * \cdots$ is then $\Lambda(y ; \psi)=\cos \left(r_{1} y\right)$ - $\cos \left(r_{2} y\right) \cdots$. The spectrum $S(\psi)$ is a bounded set or the whole $R_{x}$ according as the series $r_{1}+r_{2}+\cdots$ converges or diverges; the point spectrum $P(\psi)$ is empty and $\psi$ is either singular or absolutely continuous.

A necessary condition for the absolute continuity of $\psi$ is $\Lambda(y ; \psi) \rightarrow 0$ as $|y| \rightarrow \infty$. It is clear that $\psi$ is always of radial symmetry. We illustrate Theorem 11 by the following examples:

Example 1. $r_{n}=3^{-n}$. Here $S(\psi)$ is the Cantor null-set in $|x| \leqq \frac{1}{2}$ obtained in the usual way by successive trisections and $\psi$ is the singular function discussed by Lebesgue. We have $\Lambda(y ; \psi)=\cos (y / 3) \cos \left(y / 3^{2}\right) \cdots$, hence $\Lambda\left(2 \pi 3^{n} ; \psi\right)=\cos (2 \pi / 3) \cos \left(2 \pi / 3^{2}\right) \cdots \neq 0$ for all $n$. This is, perhaps, the simplest example of a continuous $\psi$ for which $\Lambda(y ; \psi)$ does not approach zero. With regard to this example cf. Carleman [22], pp. 223-226, Hille and Tamarkin [30].

Example 2. $r_{2 n-1}=r_{2 n}=3^{-n}$. Here $\psi$ is the convolution of the previous infinite convolution with itself. This implies that $\Lambda(y ; \psi)$ does not approach zero, hence $\psi$ is singular. The spectrum $S(\psi)$ is the interval $|x| \leqq 1$. This is, 
perhaps, the simplest example of a singular $\psi$ having an interval as spectrum.

Example 3. $r_{n}=2^{-n}$. Here $S(\psi)$ is the interval $|x| \leqq 1$ and $\psi$ is absolutely continuous, its density $D(x)$ being $=\frac{1}{2}$ for $|x|<1$ and $=0$ for $|x|>1$ so that $D(x)$ is not continuous. Placing $s(y)=(\sin y) / y$ we have

$$
\Lambda(y ; \psi)=\cos (y / 2) \cos \left(y / 2^{2}\right) \cdots=s(y) .
$$

Example 4. $r_{2 n-1}=r_{2 n}=2^{-n}$. Here $\psi$ is the convolution of the previous infinite colvolution with itself. Hence $S(\psi)$ is the interval $|x| \leqq 2$ and $\psi$ is absolutely continuous, its density $D(x)$ being $=\frac{1}{2}-\frac{1}{4}|x|$ for $|x| \leqq 2$ and $=0$ for $|x|>2$ so that $D(x)$ is continuous.

Example 5. $r_{1}, r_{2}, \cdots$ is a rearrangement of the double sequence $2^{-(l+m)}$ where $l, m=1,2, \cdots$. Here $S(\psi)$ is the interval $|x| \leqq 1$ and $\Lambda(y ; \psi)$ $=s(y / 2) s\left(y / 2^{2}\right) \cdots$ by Example 3. Hence $\Lambda(y ; \psi)$ satisfies (3.1) for every $p$ so that $\psi$ is absolutely continuous with a continuous density $D(x)$ possessing continuous derivatives of arbitrarily high order.

Example 6. $r_{1}, r_{2}, \cdots$ consists of the numbers $2^{-m !}$ where $m=1,2, \cdots$, and contains $2^{-m !}$ exactly $2^{m !}$ times. Here $r_{1}^{2}+r_{2}^{2}+\cdots$ is convergent and $r_{1}+r_{2}+\cdots$ is divergent so that $\psi$ exists and $S(\psi)=R_{x}$. It is easy to see from

$$
\Lambda\left(2 \pi 2^{m !} ; \psi\right)=\prod_{n>m}\left(\cos \left(2 \pi 2^{m !-n !}\right)\right)^{n !}
$$

that $\Lambda(y ; \psi)$ does not approach zero; hence $\psi$ is singular.

Example 7. $r_{n}=1 / n$. This is a rearrangement of the double sequence $2^{-l} /(2 m+1)$ where $l, m=0,1,2, \cdots$. Hence $\Lambda(y ; \psi)=s(y) s(y / 3) \cdots$ by Example 3 (cf.Lévy [41],p.154). Thus we have the same situation as in Example 5 except that $S(\psi)$ is now the whole $R_{x}$. Furthermore, the density $D(x)$ is regular analytic in $R_{x}$ (which cannot be the case in Example 5); in fact, $|s(y)| \leqq \min \left(1,|y|^{-1}\right)$, hence on writing $t=\frac{1}{2}|y|$ we have

$$
|\Lambda(y ; \psi)| \leqq \prod_{m=1}^{\infty} \min \left(1,(2 m-1)|y|^{-1}\right) \leqq \prod_{m=1}^{\infty} \min \left(1,2 m|y|^{-1}\right)=\prod_{m \leqq t} m t^{-1}
$$

so that $\Lambda(y ; \psi)=O\left(t^{1 / 2} e^{-t}\right)$, by Stirling's formula. It follows therefore from $t=\frac{1}{2}|y|$ that $\Lambda(y ; \psi)$ satisfies (3.2) with every $A<\frac{1}{2}$.

Example 8. $r_{n}=2^{-n} / n-2^{-(n+1)} /(n+1)$. Here $S(\psi)$ is the null-set obtained from the interval $|x| \leqq \frac{1}{2}$ by a construction identical with that leading to the Cantor null-set except that in the $n$th step the length of each of the omitted intervals is $1 /(n+1)$ times the length of each of the $2^{n-1}$ intervals obtained in the $(n-1)$ th step. The interest of this example is due to the fact that $\Lambda(y ; \psi)$ approaches zero although $\psi$ is singular. Cf. Menchoff [43].

For further results cf. Wintner [60]. 
The results of this section could also be stated in terms of the Rademacher functions.

\section{FOURIER TRANSFORMS OF DISTRIBUTIONS ON CONVEX CURVES}

Let the dimension number $k$ be 2 and let $S$ be a closed curve in $R_{x}$ given by a parametric representation $x=x(\theta)=\left(\xi_{1}(\theta), \xi_{2}(\theta)\right)$ such that $x(\theta)$ has the primitive period 1. This parametric representation of $S$ determines in $R_{x}$ a distribution function $\phi$ where $\phi(E)$ for a given Borel set $E$ is the $\theta$-measure of $E S$; we have $S(\phi)=S$. We call $\phi$ the distribution function determined by the parametric representation $x=x(\theta)$ of $S$. If $S$ is the circle $|x|=r$ given in the parametric representation $x=x(\theta)=(r \cos 2 \pi \theta, r \sin 2 \pi \theta)$ then $\phi$ is the circular equidistribution on $S$. In this case we know from $\S 5$ that $\Lambda(y ; \phi)=O\left(|y|^{-1 / 2}\right)$ as $|y| \rightarrow \infty$. We shall now prove that this appraisal holds for a general class of convex curves. In the case of a circular equidistribution, $\Lambda(y ; \phi)$ is a function of $|y|$ only; in the present case we have

$$
\Lambda(y ; \phi)=\int_{S} e^{i \sigma_{\tau}(\theta)|y|} d \theta
$$

where $y=(|y| \cos \tau,|y| \sin \tau)$ and $g_{\tau}(\theta)=\xi_{1}(\theta) \cos \tau+\xi_{2}(\theta) \sin \tau$.

THEOREM 12. Let $x=x(\theta)=\left(\xi_{1}(\theta), \xi_{2}(\theta)\right)$ be a parametric representation of a convex curve $S$, such that

(i) $\xi_{1}(\theta)$ and $\xi_{2}(\theta)$ possess continuous second derivatives $\xi_{1}^{\prime \prime}(\theta)$ and $\xi_{2}^{\prime \prime}(\theta)$;

(ii) the second derivative $g_{\tau}{ }^{\prime \prime}(\theta)$ with respect to $\theta$ of the function $g_{\tau}(\theta)$ $=\xi_{1}(\theta) \cos \tau+\xi_{2}(\theta) \sin \tau$ has for every fixed value of $\tau$ exactly two zeros $\theta$ on $S$.

Then for the Fourier transform of the distribution function $\phi$ determined by the parametric representation $x=x(\theta)$ of $S$ we have, uniformly in $\tau$,

$$
\Lambda(y ; \phi)=O\left(|y|^{-1 / 2}\right) \text { as }|y| \rightarrow \infty .
$$

The geometrical meaning of $g_{\tau}(\theta)$ implies that $g_{\tau}^{\prime}(\theta)$ has for every fixed value of $\tau$ at least two zeros on $S$; since between any two zeros of $g_{\tau}^{\prime}(\theta)$ we find at least one zero of $g_{\tau}^{\prime \prime}(\theta)$ it follows that $g_{r}^{\prime}(\theta)$ also has exactly two zeros which separate those of $g_{\tau}^{\prime \prime}(\theta)$ for every fixed $\tau$.

The zeros of $g_{\tau}^{\prime}(\theta)$ and $g_{\tau}^{\prime \prime}(\theta)$ depend continuously on $\tau$. For if $\tau_{n} \rightarrow \tau$, every limit point of zeros of $g_{\tau_{n}}{ }^{\prime}(\theta)$ or $g_{\tau_{n}}{ }^{\prime \prime}(\theta)$ will, by the continuity of $g_{\tau}{ }^{\prime}(\theta)$ and $g_{\tau}{ }^{\prime \prime}(\theta)$ as functions of $\theta$ and $\tau$ together, be a zeroof $g_{\tau}{ }^{\prime}(\theta)$ or $g_{\tau}{ }^{\prime \prime}(\theta)$. Furthermore, the two zeros of $g_{\tau_{n}}{ }^{\prime}(\theta)$ cannot tend to the same point on $S$ as $n \rightarrow \infty$, for then this point would also be a limit point of zeros of $g_{\tau_{n}}{ }^{\prime \prime}(\theta)$, hence $g_{\tau}^{\prime}(\theta)$ and $g_{\tau}^{\prime \prime}(\theta)$ would have a common zero, which is, as we saw, impossible. The same argument shows that the two zeros of $g_{\tau_{n}}{ }^{\prime \prime}(\theta)$ cannot tend to the same point on $S$ as $n \rightarrow \infty$. 
We now consider, for every fixed $\tau$, the mid-points of the four arcs on $S$ determined by the zeros of $g_{\tau}^{\prime}(\theta)$ and $g_{\tau}^{\prime \prime}(\theta)$; these mid-points also depend continuously on $\tau$ and divide $S$ into four arcs $A_{\tau}, B_{\tau}, C_{\tau}, D_{\tau}$ such that $A_{\tau}$ and $C_{\tau}$ each contain one of the zeros of $g_{\tau}^{\prime}(\theta)$ and $B_{\tau}$ and $D_{\tau}$ each contain one of the zeros of $g_{\tau}^{\prime \prime}(\theta)$. Since the end points of $A_{\tau}, B_{\tau}, C_{\tau}, D_{\tau}$ depend continuously on $\tau$ there exists a constant $\alpha>0$ such that

$$
\left|g_{\tau}^{\prime \prime}(\theta)\right| \geqq \alpha \text { on } A_{\tau} \text { and } C_{\tau} \text { and }\left|g_{\tau}^{\prime}(\theta)\right| \geqq \alpha \text { on } B_{\tau} \text { and } D_{\tau} .
$$

Now if $f(\theta), \gamma \leqq \theta \leqq \delta$, is a real-valued function possessing a continuous monotone derivative $f^{\prime}(\theta)$ which is nowhere zero, then

$$
\left|\int_{\gamma}^{\delta} e^{i f(\theta)} d \theta\right| \leqq 4 / \min \left|f^{\prime}(\theta)\right| .
$$

Furthermore, if $f(\theta), \gamma \leqq \theta \leqq \delta$, is a real-valued function possessing a continuous second derivative $f^{\prime \prime}(\theta)$ which is nowhere zero, then

$$
\left|\int_{\gamma}^{\delta} e^{i f(\theta)} d \theta\right| \leqq 8 / \min \left|f^{\prime \prime}(\theta)\right|^{1 / 2}
$$

The inequality (7.3) follows from the identity

$$
\left|\int_{\gamma}^{\delta} e^{i f(\theta)} d \theta\right|=\left|\int_{\gamma}^{\delta} \frac{\left(e^{i f(\theta)}\right)^{\prime}}{f^{\prime}(\theta)} d \theta\right|
$$

in virtue of the second mean-value theorem and (7.4) is, according to van der Corput and Landau, a consequence of (7.3); cf. Landau [39], p. 60.

On applying (7.3) and (7.4) to the four integration domains $A_{\tau}, B_{\tau}, C_{\tau}, D_{\tau}$ and to the function $f(\theta)=g_{\tau}(\theta)|y|$, it follows from (7.1) and (7.2) that

$$
|\Lambda(y ; \phi)| \leqq 16 \alpha^{-1 / 2}|y|^{-1 / 2}+16 \alpha^{-1}|y|^{-1},
$$

which proves Theorem 12. Since $\alpha^{-1}|y|^{-1} \leqq \alpha^{-1 / 2}|y|^{-1 / 2}$ when $\alpha|y| \geqq 1$, and $|\Lambda(y ; \phi)| \leqq 1$, we find also

$$
|\Lambda(y ; \phi)| \leqq 32 \alpha^{-1 / 2}|y|^{-1 / 2}
$$

Let $F(z)=a_{1} z+a_{2} z^{2}+\cdots$ be a power series convergent in a circle $|z|<\rho$ $(\leqq \infty)$ and such that $a_{1} \neq 0$. Let $S$ denote the curve $x=x(\theta)=\xi_{1}(\theta)+i \xi_{2}(\theta)$ $=F\left(r e^{2 \pi i \theta}\right)$ where $0<r<\rho$ and let $\phi$ be the distribution function in $R_{x}$ determined by this parametric representation of $S$. Then $c(\phi)=0$, i.e., $\phi=\tilde{\phi}$, and $\mathrm{M}_{2}(\phi)=\left|a_{1}\right|^{2} r^{2}+\left|a_{2}\right|^{2} r^{4}+\ldots$. It is known that $S$ is a convex curve if $r$ is sufficiently small; condition (i) of Theorem 12 is satisfied for all $r$; finally if $a_{n}=\left|a_{n}\right| e^{2 \pi i \gamma_{n}}$ we have 
(7.6) $g_{\tau}(\theta)=\left|a_{1}\right| r \cos 2 \pi\left(\theta+\gamma_{1}-\tau\right)+\left|a_{2}\right| r^{2} \cos 2 \pi\left(2 \theta+\gamma_{2}-\tau\right)+\cdots$,

showing that if $r$ is sufficiently small then condition (ii) of Theorem 12 also is satisfied. Hence there exists a positive $\rho_{0}(\leqq \rho)$ such that all conditions of Theorem 12 are satisfied for $0<r<\rho_{0}$. Finally, there exists for any given $\rho_{1}<\rho_{0}$ a constant $c>0$ such that the number $\alpha>0$ defined by (7.2) and occurring in (7.5) may be chosen $>c r$ if $0<r \leqq \rho_{1}$. This is clear from (7.6) if $\rho_{1}$ is sufficiently small; on the other hand if $\rho_{1}<\rho_{0}$ and $\epsilon>0$ are arbitrary then the same argument which was applied in the proof of Theorem 12 shows that the zeros of $g_{r}^{\prime}(\theta)$ and $g_{r}^{\prime \prime}(\theta)$ depend continuously on $\tau$ and $r$ together if $\epsilon \leqq r \leqq \rho_{1}$. On collecting the results we have

THEOREM 13. Let $F(z)=a_{1} z+a_{2} z^{2}+\cdots$, where $a_{1} \neq 0$, be convergent for $|z|<\rho(\leqq \infty)$. Let $S$ denote the curve $x=x(\theta)=F\left(r e^{2 \pi i \theta}\right)$ where $0<r<\rho$, and let $\phi$ be the distribution function in $R_{x}$ determined by this parametric representation of $S$. Then there exists a positive $\rho_{0}(\leqq \rho)$ such that $S$ is convex and $\Lambda(y ; \phi)$ $=O\left(|y|^{-1 / 2}\right)$ if $0<r<\rho_{0}$. Furthermore, if $0<\rho_{1}<\rho_{0}$, there exists a constant $\beta$ such that

$$
\left.\Lambda(y ; \phi)\left|\leqq \beta r^{-1 / 2}\right| y\right|^{-1 / 2}
$$

if $0<r \leqq \rho_{1}$.

\section{A TYPE OF INFINITE CONVOLUTION}

As an application of previous results we prove

THEOREM 14. Lel $F(z)=a_{1} z+a_{2} z^{2}+\cdots$, where $a_{1} \neq 0$, be convergent for $|z|<\rho(\leqq \infty)$. Let $r<\rho$ be given and let $r_{1}, r_{2}, \cdots$ be a sequence of positive numbers such that $r_{n} \leqq r$ for all $n$. Let $S_{n}$ denote the curve $x=x_{n}(\theta)=F\left(r_{n} e^{2 \pi i \theta}\right)$ and $\phi_{n}$ the distribution function in $R_{x}$ determined by this parametric representation of $S_{n}$. Then the convergence of $r_{1}^{2}+r_{2}^{2}+\cdots$ is necessary and sufficient for both the convergence and the absolute convergence of $\phi_{1} * \phi_{2} * \ldots$. The spectrum $S(\psi)$ of $\psi=\phi_{1} * \phi_{2} * \cdots$ is then either a bounded set or the whole $R_{x}$ according as $r_{1}+r_{2}+\cdots$ is convergent or divergent. The distribution function $\psi$ is always absolutely continuous with a continuous density $D(x)=D\left(\xi_{1}, \xi_{2}\right)$ possessing continuous partial derivatives of arbitrarily high order. Finally $D(x)>0$ for all $x$ if $r_{1}+r_{2}+\cdots$ diverges.

Since $r_{n} \leqq r<\rho$, all $S\left(\phi_{n}\right)$ are contained in a sufficiently large circle $|x| \leqq K$. Furthermore, $c\left(\phi_{n}\right)=0$ so that Theorems 5 and 6 give as a necessary and sufficient condition for both the convergence and the absolute convergence of $\phi_{1} * \phi_{2} * \cdots$ the convergence of the series

$$
\sum_{n=1}^{\infty} \mathrm{M}_{2}\left(\phi_{n}\right)=\sum_{n=1}^{\infty} \sum_{m=1}^{\infty}\left|a_{m}\right|^{2} r_{n}{ }^{2 m} \text {. }
$$


This proves the first part of the theorem. Since $r<\rho$, there exists a constant $C$ such that $|F(z)| \leqq C|z|$ and $\left|F(z)-a_{1} z\right| \leqq C|z|^{2}$ for $|z| \leqq r$. Hence if $r_{1}+r_{2}+\cdots$ converges, $S(\psi)$ is a bounded set. Suppose now that $r_{1}+r_{2}+\cdots$ diverges and let $x_{0}$ be any point of $R_{x}$. For a given $\epsilon>0$, let $p$ be so large that $r_{p+1}^{2}+r_{p+2}^{2}+\cdots<\epsilon / C$. Let $x_{1}$ be an arbitrary point of the form $F\left(r_{1} e^{2 \pi i \theta_{1}}\right)$ $+\cdots+F\left(r_{p} e^{2 \pi i \theta_{p}}\right)$; then if $n>p$ is large enough we have $\left|a_{1}\right|\left(r_{p+1}+\cdots+r_{n}\right)$ $>\left|x_{0}-x_{1}\right|$ and none of the numbers $r_{p+1}, \cdots, r_{n}$ is larger than the sum of the $n-p-1$ others. As in Theorem 9 we may therefore choose $\theta_{p+1}, \cdots, \theta_{n}$ such that $a_{1}\left(r_{p+1} e^{2 \pi i \theta_{p+1}}+\cdots+r_{n} e^{2 \pi i \theta_{n}}\right)=x_{0}-x_{1}$, implying that $\mid F\left(r_{1} e^{2 \pi i \theta_{1}}\right)$ $+\cdots+F\left(r_{n} e^{2 \pi i \theta_{n}}\right)-x_{0} \mid<\epsilon$. Hence the circle $\left|x-x_{0}\right|<\epsilon$ contains for sufficiently large $n$ points of $S_{1}+\cdots+S_{n}$, which means by Theorem 3 that $S(\psi)$ contains the arbitrary point $x_{0}$. Since $r_{n} \rightarrow 0$ it follows from Theorem 13 that $\Lambda(y ; \psi)$ satisfies (3.1) for all $p$. For the proof of the last statement of Theorem 14, cf. the Remark following Theorem 9.

Remark. An analogous reasoning shows that if $r_{n} \rightarrow 0$ then $\psi_{n}=\phi_{1} * \ldots$ $* \phi_{n}$ is absolutely continuous with a continuous density $D_{n}(x)$ if $n>n_{0}$ and $D_{n}(x)=D_{n}\left(\xi_{1}, \xi_{2}\right)$ possesses continuous partial derivatives of order $\leqq p$ if $n>n_{p}$. If $r_{1}^{2}+r_{2}^{2}+\cdots$ converges then $D_{n}(x)$ and its partial derivatives converge uniformly to $D(x)$ and its partial derivatives, whereas they tend uniformly to zero as $n \rightarrow \infty$ if $r_{1}^{2}+r_{2}^{2}+\cdots$ diverges; for a proof cf. the Remark following Theorem 8 .

If $r_{1}+r_{2}+\cdots$ is convergent then the density $D(x)=D\left(\xi_{1}, \xi_{2}\right)$ cannot be regular analytic in every point of the real plane $R_{x}$ since $D(x) \equiv 0$ outside of the bounded set $S(\psi)$. We have, however, the following theorem:

TheOREM 15. If $r_{n}^{-1}=O(n)$, then the density $D(x)=D\left(\xi_{1}, \xi_{2}\right)$ defined in Theorem 14 is regular analytic in every point of the real plane $R_{x}$. If $r_{n}^{-1}=o(n)$, then $D(x)$ is an entire function of the two variables $\xi_{1}, \xi_{2}$.

Let $\rho_{1}<\rho_{0}$ be fixed and let $\beta$ denote the corresponding constant defined in Theorem 13. Suppose first that all $r_{n} \leqq \rho_{1}$. We have then $\left|\Lambda\left(y ; \phi_{n}\right)\right|$ $\leqq \beta r_{n}^{-1 / 2}|y|^{-1 / 2}$ for every $n$. Since $r_{n}^{-1} \leqq a n$ for some $a>0$ and $\mid \Lambda(y$; $\left.\phi_{n}\right) \mid \leqq 1$, it follows that

$$
\left|\Lambda\left(y ; \phi_{n}\right)\right| \leqq \min \left(1, \beta a^{1 / 2} n^{1 / 2}|y|^{-1 / 2}\right) .
$$

Thus on placing $t=\beta^{-2} a^{-1}|y|$ we have

$$
\left|\Lambda\left(y ; \phi_{n}\right)\right| \leqq \min \left(1, n^{1 / 2} t^{-1 / 2}\right) .
$$

It follows therefore from 
that

$$
|\Lambda(y ; \psi)|=\prod_{n=1}^{\infty}\left|\Lambda\left(y ; \phi_{n}\right)\right| \leqq \prod_{n \leqq t}\left|\Lambda\left(y ; \phi_{n}\right)\right|
$$

$$
|\Lambda(y ; \psi)| \leqq \prod_{n \leqq t} \min \left(1, n^{1 / 2} t^{-1 / 2}\right)=\prod_{n \leqq t} n^{1 / 2} t^{-1 / 2}
$$

for every $t$. Hence $\Lambda(y ; \psi)=O\left(t^{1 / 4} e^{-t / 2}\right)$, by Stirling's formula. We see therefore from $t=\beta^{-2} a^{-1}|y|$ that $\Lambda(y ; \psi)$ satisfies (3.2) with every $A<\frac{1}{2} \beta^{-2} a^{-1}$. Let us now drop the assumption $r_{n} \leqq \rho_{1}$ and let $b=\lim \sup _{n \rightarrow \infty} r_{n}^{-1} / n$. Then if $a>b$ there exists an $n_{0}$ such that $r_{n} \leqq \rho_{1}$ and $r_{n}^{-1} \leqq a\left(n-n_{0}\right)$ for all $n>n_{0}$. Hence $\Lambda(y ; \psi)$ satisfies again (3.2) with every $A<\frac{1}{2} \beta^{-2} a^{-1}$, which proves the first part of the theorem. If $b=0$ we may take $a$ arbitrarily small; hence $\Lambda(y ; \psi)$ satisfies (3.2) for arbitrarily large $A$ which proves the second part of the theorem.

Returning to the case of general $r_{n}$ we shall now give an appraisal for the density $D(x)$ occurring in Theorem 14 .

THEOREM 16. For any $\lambda>0$ the density $D(x)$ defined in Theorem 14 is $=O\left(e^{-\lambda|x|^{2}}\right)$ as $|x| \rightarrow \infty$ and each of its partial derivatives also is $=O\left(e^{-\lambda|x|^{2}}\right)$ as $|x| \rightarrow \infty$.

The proof is based upon an argument of Paley and Zygmund [44]. Let $\lambda$ be fixed, let $q$ be a fixed positive integer so large that

$$
d=1-2 \lambda\left|a_{1}\right|^{2}\left(r_{q+1}^{2}+r_{q+2}^{2}+\cdots\right)
$$

is positive, and let $n$ be a variable integer $>q$. Placing

$$
s_{n}\left(\theta_{1}, \cdots, \theta_{n}\right)=F\left(r_{1} e^{2 \pi i \theta_{1}}\right)+\cdots+F\left(r_{n} e^{2 \pi i \theta_{n}}\right)
$$

and

$$
t_{n}\left(\theta_{q+1}, \cdots, \theta_{n}\right)=a_{1} r_{q+1} e^{2 \pi i \theta_{q+1}}+\cdots+a_{1} r_{n} e^{2 \pi i \theta_{n}}
$$

we have

$$
\left|s_{n}\left(\theta_{1}, \cdots, \theta_{n}\right)-t_{n}\left(\theta_{q+1}, \cdots, \theta_{n}\right)\right| \leqq A
$$

where $A$ is a constant independent of $n$ in virtue of the convergence of $r_{1}^{2}+r_{2}^{2}+\cdots$. Hence from $\left|s_{n}\right|^{2} \leqq 2\left|s_{n}-t_{n}\right|^{2}+2\left|t_{n}\right|^{2}$

$$
\int_{c_{n}} d \theta_{n} \cdots \int_{c_{1}} e^{\lambda\left|\theta_{n}\left(\theta_{1}, \cdots, \theta_{n}\right)\right|^{2}} d \theta_{1} \leqq e^{2 \lambda \Lambda^{2}} \int_{c_{n}} d \theta_{n} \cdots \int_{c_{q+1}} e^{2 \lambda\left|t_{n}\left(\theta_{q+1}, \cdots, \theta_{n}\right)\right|^{2}} d \theta_{q+1}
$$

where $c_{\nu}$ denotes the circle of length 1 on which $\theta_{\nu}$ is the variable point. Now it is known (cf., for a detailed proof, Jessen [35], pp. 290-291) that the integral on the right is 
so that

$$
\leqq\left(1-2 \lambda\left(\left|a_{1}\right|^{2} r_{q+1}^{2}+\cdots+\left|a_{1}\right|^{2} r_{n}^{2}\right)\right)^{-1} \leqq d^{-1}
$$

$$
\int_{c_{n}} d \theta_{n} \cdots \int_{c_{1}} e^{\lambda\left|\delta_{n}\left(\theta_{1}, \cdots, \theta_{n}\right)\right|^{2}} d \theta_{1} \leqq K
$$

where $K=e^{2 \lambda \Lambda^{2}} d^{-1}$. Placing $h(x)=e^{\lambda|x|^{2}}$ in (2.2) and using the relation

$$
\int_{R_{x_{n}}} \phi_{n}\left(d R_{x_{n}}\right) \cdots \int_{R_{x_{1}}} e^{\lambda\left|x_{1}+\cdots+x_{n}\right|^{2} \phi_{1}}\left(d R_{x_{1}}\right)=\int_{c_{n}} d \theta_{n} \cdots \int_{c_{1}} e^{\lambda\left|s_{n}\left(\theta_{1}, \cdots, \theta_{n}\right)\right|^{2}} d \theta_{1},
$$

which is clear from the definition of $\phi_{\nu}$, one obtains

$$
\int_{R_{x}} e^{\lambda|x|^{2}} \psi_{n}\left(d R_{x}\right) \leqq K
$$

It follows therefore from (2.1) by letting $n \rightarrow \infty$ that

$$
\int_{R_{x}} e^{\lambda|x| 2} \psi\left(d R_{x}\right) \leqq K
$$

Consequently if $E$ is a bounded Borel set in $R_{x}$ then $\psi(x-E)=O\left(e^{-\lambda|x|^{2}}\right)$ as $|x| \rightarrow \infty$; in fact, if $E$ lies in the circle of radius $r$ about the origin then $e^{\lambda(|x|-r)^{2}} \psi(x-E) \leqq K$ if $|x|>r$. Similarly, if $n$ is arbitrary and $\rho_{n}=\phi_{n+1}$ $* \phi_{n+2} * \cdots$ then $\rho_{n}(x-E)=O\left(e^{-\lambda|x|^{2}}\right)$ as $|x| \rightarrow \infty$ for any bounded Borel set $E$. Now $\psi_{n}$ is by the Remark following the proof of Theorem 14 absolutely continuous with a continuous density $D_{n}(x)$ if $n>n_{0}$; hence from $\psi=\psi_{n} * \rho_{n}$

$$
D(x)=\int_{R_{u}} D_{n}(x-u) \rho_{n}\left(d R_{u}\right),
$$

so that $D(x) \leqq M_{n} \rho_{n}\left(x-S\left(\psi_{n}\right)\right)$ where $M_{n}$ is the maximum of $D_{n}(x)$. Since $S\left(\psi_{n}\right)$ is a bounded set it follows that $D(x)=O\left(e^{-\lambda|x|^{2}}\right)$ as $|x| \rightarrow \infty$. Also, if $p>0$ is given, $D_{n}(x)$ has continuous partial derivatives of order $\leqq p$ for $n>n_{p}$ and the corresponding partial derivatives of $D(x)$ may be obtained from (8.1) by differentiation under the integral sign. This implies that each partial derivative of $D(x)$ is $=O\left(e^{-\lambda|x|^{2}}\right)$ as $|x| \rightarrow \infty$.

Remark. Theorem 16 implies that $\mathbf{M}_{p}(\psi)$ is finite for every $p$ so that all moments $\mu_{q_{1}, q_{2}}(\psi)$ of $\psi$ exist; furthermore, these moments belong to a $d e-$ termined moment problem (cf. Haviland [29]). This remark applies in particular to the infinite convolution $\psi_{\sigma}$ occurring in Theorem 19.

\section{VECTORIAL ADDITION OF CONVEX CURVES}

In order to determine the spectrum $S(\psi)$ of the distribution function $\psi=\phi_{1} * \phi_{2} * \cdots$, occurring in Theorem 14 , in the case where $r_{1}+r_{2}+\cdots$ 
converges and all $S_{n}$ are convex, we first prove a theorem concerning the vectorial sum of $n$ convex curves. If $C$ is a convex curve we shall denote by $I(C)$ and $E(C)$ the two open domains into which $C$ divides $R_{x}$, such that $I(C)$ is the interior and $E(C)$ the exterior domain, and we shall denote by $I(C)$ and $\bar{E}(C)$ the closures of $I(C)$ and $E(C)$.

TheOREM 17. If $S_{1}, \cdots, S_{n}$ are $n$ convex curves, then their vectorial sum $T_{n}=S_{1}+\cdots+S_{n}$ is either the closed convex domain $I\left(B_{n}\right)$ determined by $a$ convex curve $B_{n}$ or it is a ring-shaped domain, namely a closed convex domain $I\left(B_{n}\right)$ minus an open convex domain $I\left(A_{n}\right)$.

For $n=1$ the theorem is true with $B_{1}=A_{1}=S_{1}$. Suppose that it is true for $n$ and let us prove it for $n+1$. The complementary set $T_{n}^{\prime}$ of $T_{n}$ consists either of a single domain $E\left(B_{n}\right)$ or of two domains $E\left(B_{n}\right)$ and $I\left(A_{n}\right)$. Now in order that a point $x$ shall belong to the complementary set $T_{n+1}{ }^{\prime}$ of $T_{n+1}$ it is necessary and sufficient that the curve $x-S_{n+1}$ belongs to $T_{n}^{\prime}$. This may happen in three different ways:

(i) it may belong to $I\left(A_{n}\right)$;

(ii) it may belong to $E\left(B_{n}\right)$ and contain $B_{n}$ in its interior;

(iii) it may belong to $E\left(B_{n}\right)$ without containing $B_{n}$ in its interior.

(i) occurs only when $A_{n}$ exists; it means that $I\left(x-S_{n+1}\right)$ is contained in $I\left(A_{n}\right)$; (ii) means that $\bar{E}\left(x-S_{n+1}\right)$ is contained in $E\left(B_{n}\right)$ and (iii) means that $I\left(x-S_{n+1}\right)$ is contained in $E\left(B_{n}\right)$. There are always points $x$ for which (iii) occurs whereas (i) and (ii) need not occur and are mutually exclusive. Hence it is sufficient to prove that the set of points $x$ for which (iii) occurs is of the type $E\left(B_{n+1}\right)$ where $B_{n+1}$ is a convex curve and that the set of points $x$ for which (i) or (ii) occurs (if such points exist) is of the type $I\left(A_{n+1}\right)$ where $A_{n+1}$ is a convex curve. This is trivial as far as (i) and (ii) are concerned; in fact if (i) is true for both $x=x_{1}$ and $x=x_{2}$ it is obviously true for any $x$ on the segment $x_{1} x_{2}$ and the same holds for (ii). We therefore only need to consider the case (iii). The complementary set $H$ to the set of points $x$ for which (iii) occurs is the set of points for which $I\left(x-S_{n+1}\right)$ and $I\left(B_{n}\right)$ have at least one point in common. Now $I\left(x-S_{n+1}\right)=x-I\left(S_{n+1}\right)$. Hence $H$ is simply the vectorial sum of the two closed convex domains $I\left(B_{n}\right)$ and $I\left(S_{n+1}\right)$ which is known to be a closed convex domain $I\left(B_{n+1}\right)$. This completes the proof of Theorem 17 .

THEOREM 18. If $S_{1}, S_{2}, \cdots$ are convex curves surrounding the origin such that the diameter $d_{n}$ of $S_{n}$ tends to zero as $n \rightarrow \infty$ then the vectorial sum $T=S_{1}$ $+S_{2}+\cdots$ is a bounded set if and only if the series $d_{1}+d_{2}+\cdots$ is convergent and $T$ is then either a closed convex domain $\bar{I}(B)$ or a ring-shaped domain, namely a closed convex domain $I(B)$ minus an open convex domain $I(A)$. 
With the previous notation it is clear from the proof of Theorem 17 that the curves $B_{1}, B_{2}, \cdots$ all surround the origin and that $B_{n+1}$ surrounds $B_{n}$. Hence, if $n$ is sufficiently large $B_{n}$ surrounds $S_{n+1}$ so that the possibility (ii) is excluded. This implies that from a certain $n$ on the existence of $A_{n}$ is necessary for the existence of $A_{n+1}$ which is then surrounded by $A_{n}$. Since the diameters of the curves $B_{n}$ remain bounded if and only if the series $d_{1}+d_{2}+\cdots$ is convergent, Theorem 18 follows from $T=\lim T_{n}$.

Remark. The supporting function (Stützfunktion) of $B$ is the sum of the supporting functions of $S_{1}, S_{2}, \cdots$. There is no corresponding rule for the supporting function of $A$ and there is not even a simple rule enabling us to decide whether $A$ exists. If, however, one of the given curves, say $S_{1}$, surrounds all the others we have the rule that $A$ exists if and only if there exist points $x$ such that $x-\left(S_{2}+S_{3}+\cdots\right)$ belongs to $I\left(S_{1}\right)$ and these $x$ form then the domain $I(A)$.

Theorem 18 applies in particular to the spectrum $S(\psi)$ of the infinite convolution $\psi=\phi_{1} * \phi_{2} * \cdots$, occurring in Theorem 14, in the case where $r_{1}+r_{2}+\cdots$ is convergent and every $S\left(\phi_{n}\right)$ is convex.

The problem considered in this section has been studied in greater detail by Bohr [7]; cf. also Bohr and Jessen [19] and Haviland [26].

10. Distribution FUNCTIONS OCCURRING IN THE THEORY OF THE ZETA FUNCTION

For later application we consider the case $F(z)=z+\frac{1}{2} z^{2}+\cdots$ $=-\log (1-z)$ where $|z|<1$. The curve $S$ defined by the parametric representation $x=x(\theta)=\xi_{1}(\theta)+i \xi_{2}(\theta)=-\log \left(1-r e^{2 \pi i \theta}\right)$, where $0<r<1$, is convex since the angle between the tangent of $S$ at the point $\theta$ and a horizontal line through this point is, in virtue of the conformity, equal to the angle between the tangent of the circle $z=1-r e^{2 \pi i \theta}$ at the point $\theta$ and the line joining the origin with this point. Furthermore, if $\tau$ is arbitrary, the function $g_{r}^{\prime \prime}(\theta)$ has exactly two zeros $\theta$ on $S$ since $g_{\tau}^{\prime \prime}(\theta)$ is a trigonometrical polynomial in $2 \pi \theta$ of the first order multiplied by a non-vanishing factor. Thus the number $\rho_{0}$ defined in Theorem 13 is $=1$. It may be mentioned that $S$ surrounds the origin and that its diameter $d$ tends to zero as $r \rightarrow 0$. Also, the distribution function determined by the parametric representation of $S$ is symmetrical with respect to the line $\xi_{2}=0$. Applying the results of $\S 8$ and $\S 9$ we prove the following theorem:

THEOREM 19. Let $p_{1}, p_{2}, \cdots$ denote the prime numbers $2,3, \cdots$, and let $\sigma>0$ be fixed. Let $S_{n, \sigma}$ denote the curve $x=x_{n, \sigma}=-\log \left(1-p_{n}{ }^{-\sigma} e^{2 \times i \theta}\right)$ and $\phi_{n, \sigma}$ the distribution function determined by this parametric representation of $S_{n, \sigma}$. Then the infinite convolution $\phi_{1, \sigma} * \phi_{2, \sigma} * \cdots$ is convergent and also absolutely 
convergent if and only if $\sigma>\frac{1}{2}$, and $\psi_{\sigma}=\phi_{1, \sigma} * \phi_{2, \sigma} * \cdots$ is then symmelric with respect to the line $\xi_{2}=0$. If $\frac{1}{2}<\sigma \leqq 1$, the spectrum $S\left(\psi_{\sigma}\right)$ is $=R_{x}$; if $\sigma>1$, it is either a closed convex domain $I\left(B_{\sigma}\right)$ or a ring-shaped domain, namely a closed convex domain $I\left(B_{\sigma}\right)$ minus an open convex domain $I\left(A_{\sigma}\right)$. The distribution function $\psi_{\sigma}$ is always absolutely continuous and its density $D_{\sigma}(x)=D_{\sigma}\left(\xi_{1}, \xi_{2}\right)$ is continuous and possesses continuous partial derivatives of all orders. If $\frac{1}{2}<\sigma \leqq 1$, then $D_{\sigma}(x)>0$ for all $x$. For any fixed $\lambda>0$ we have $D_{\sigma}(x)=O\left(e^{-\lambda|x|^{2}}\right)$ as $|x| \rightarrow \infty$ and every partial derivative of $D_{\sigma}(x)$ also is $=O\left(e^{-\lambda|x|^{2}}\right)$ as $|x| \rightarrow \infty$. Finally, if $\frac{1}{2}<\sigma<1$, then $D_{\sigma}(x)$ is an entire function of the two variables $\xi_{1}, \xi_{2}$.

The first part of the theorem follows from Theorem 14 since $\sigma>\frac{1}{2}$ is necessary and sufficient for the convergence of $p_{1}{ }^{-3 \sigma}+p_{2}^{-2 \sigma}+\cdots$. The symmetry of $\psi_{\sigma}$ with respect to the line $\xi_{2}=0$ is obvious. Since the condition $\sigma>1$ is necessary and sufficient for the convergence of $p_{1}^{-\sigma}+p_{2}^{-\sigma}+\cdots$, it follows from Theorem 14 that $S\left(\psi_{\sigma}\right)$ is $=R_{x}$ if $\sigma \leqq 1$ and is a bounded set if $\sigma>1$; the description of $S\left(\psi_{\sigma}\right)$ for $\sigma>1$ follows from Theorem 18. The absolute continuity of $\psi_{\sigma}$ and the properties of $D_{\sigma}(x)$ including the appraisals follow from Theorems 14 and 16. Finally, the last part of the theorem follows from Theorem 15 since $p_{n}{ }^{\sigma}=o(n)$ for every $\sigma<1$ (this is an elementary property of the prime numbers).

It is clear that $D_{\sigma}(x)$ is not regular analytic in every point of $R_{x}$ if $\sigma>1$; we do not know what is the situation if $\sigma=1$.

Remark. By means of the Remark following the proof of Theorem 18 it is easy to obtain a more detailed description of $S\left(\psi_{\sigma}\right)$ for $\sigma>1$. The set $I\left(B_{\sigma}\right)$ always contains the origin; it decreases when $\sigma$ increases and the limits of $I\left(B_{\sigma}\right)$ for $\sigma \rightarrow 1$ and $\sigma \rightarrow \infty$ are $R_{x}$ and the point $x=0$ respectively. Furthermore, $A_{\sigma}$ does not exist if $\sigma$ is sufficiently near to 1 , say $\sigma \leqq \sigma_{1}$, while $A_{\sigma}$ exists if $\sigma$ is sufficiently large, say $\sigma>\sigma_{2}$. It is not known whether or not $\sigma_{1}=\sigma_{2}$. Finally, $S\left(\psi_{\sigma}\right)$ is symmetric not only with respect to the line $\xi_{2}=0$ but also with respect to the line $\xi_{1}=-\frac{1}{2} \log \left(1-p_{1}^{-2 \sigma}\right)-\frac{1}{2} \log \left(1-p_{2}^{-2 \sigma}\right)-\cdots=\frac{1}{2} \log \zeta(2 \sigma)$; this line is not a symmetry axis of $\psi_{\sigma}$.

The explicit expression for the density $D_{\sigma}(x)$ as given by the inversion formula enables us to discuss $D_{\sigma}(x)$ as a function of $\sigma$. We give only one result in this direction:

THEOREM 20. The function $D_{\sigma}(x)$ and each of its partial derivatives tend uniformly to zero as $\sigma \rightarrow \frac{1}{2}$.

The Fourier transforms $\Lambda\left(y ; \psi_{\sigma}\right)$ satisfy (3.1) uniformly for $\frac{1}{2}<\sigma<\sigma_{0}$ where $\sigma_{0}>\frac{1}{2}$ is arbitrary but fixed. It is therefore sufficient to prove that $\Lambda\left(y ; \psi_{\sigma}\right) \rightarrow 0$ as $\sigma \rightarrow \frac{1}{2}$ uniformly for $|y| \geqq \epsilon$ where $\epsilon>0$ is arbitrary and this is a 
simple consequence of the divergence of $p_{1}^{-1}+p_{2}^{-1}+\cdots$. Cf. the Remark following Theorem 8 .

Remark. It may be mentioned that since $\rho_{0}=1$, the distribution function $\psi_{n, \sigma}=\phi_{1, \sigma} * \cdots * \phi_{n, \sigma}$ is for every $\sigma>0$ absolutely continuous with a continuous density $D_{n, \sigma}(x)$ whenever $n>4$, and $D_{n, \sigma}(x)=D_{n, \sigma}\left(\xi_{1}, \xi_{2}\right)$ possesses continuous partial derivatives of order $\leqq p$ whenever $n>4+2 p$. If $\sigma>\frac{1}{2}$ then $D_{n, \sigma}(x)$ and its partial derivatives tend uniformly to $D_{\sigma}(x)$ and its partial derivatives, whereas they tend uniformly to zero as $n \rightarrow \infty$ if $0<\sigma \leqq \frac{1}{2}$.

Let $x=\xi_{1}+i \xi_{2}$ and let $R_{x}$ be mapped on itself by the transformation $e^{x}$; every point $x \neq 0$ is then the image of the enumerable set of points $\log x$. If $E$ is an arbitrary set in $R_{x}$ we denote by $e^{E}$ the set of all points $e^{x}$ where $x$ belongs to $E$ and by $\log E$ the set of all points $x$ such that $e^{x}$ belongs to $E$; we notice that $\log \left(e^{E}\right)$ is not necessarily $E$. If $E$ is a Borel set, then so are the sets $e^{E}$ and $\log E$. If $\phi$ is a distribution function in $R_{x}$, a new distribution function $\bar{\phi}$ is defined by the relation $\bar{\phi}(E)=\phi(\log E)$; the spectrum $S(\bar{\phi})$ is the closure of $e^{S(\phi)}$. The set $\log E$ being a null-set if and only if $E$ is a null-set, the absolute continuity of one of the distribution functions $\phi$ and $\bar{\phi}$ implies that of the other. If $D(x)$ and $\bar{D}(x)$ denote the densities of $\phi$ and $\bar{\phi}$ and if we write

$$
\Delta(x)=\sum_{\mu=-\infty}^{\infty} D(x+2 \pi i \mu)
$$

we have $\bar{D}(x)=|x|^{-2} \Delta(\log x)$; it does not matter that in this expression $x=0$ is excluded, $D(x)$ and $\bar{D}(x)$ being determined only up to null-functions. We have now the following theorem:

THEOREM 21. Let $\bar{\psi}_{\sigma}$ be the distribution function defined by $\bar{\psi}_{\sigma}(E)=\psi_{\sigma}(\log E)$, where $\sigma>\frac{1}{2}$ and $\psi_{\sigma}$ denotes the distribution function defined in Theorem 19. Then $\bar{\psi}_{\sigma}$ is symmetric with respect to the line $\xi_{2}=0$. If $\frac{1}{2}<\sigma \leqq 1$ the spectrum $S\left(\psi_{\sigma}\right)$ is $=R_{x}$; if $\sigma>1$ then $S\left(\bar{\psi}_{\sigma}\right)=e^{S\left(\psi_{\sigma}\right)}$ which is a closed bounded set not containing $x=0$. The distribution function $\bar{\psi}_{\sigma}$ is always absolutely continuous and its density $\bar{D}_{\sigma}(x)$ is continuous and possesses continuous partial derivatives of any order. For $\frac{1}{2}<\sigma \leqq 1$ we have $\bar{D}_{\sigma}(x)>0$ for all $x \neq 0$, while $\bar{D}_{\sigma}(0)=0$. Furthermore, if $\lambda>0$ is arbitrary then $\bar{D}_{\sigma}(x)=O\left(e^{-\lambda(\log |x|)^{2}}\right)$ as $|x| \rightarrow \infty$ or $|x| \rightarrow 0$, and every partial derivative of $\bar{D}_{\sigma}(x)$ also is $=O\left(e^{-\lambda(\log |x|)^{2}}\right)$ as $|x| \rightarrow \infty$ or $|x| \rightarrow 0$. Finally, if $\frac{1}{2}<\sigma<1$ then $\bar{D}_{\sigma}\left(e^{x}\right)$ is an entire function of the two variabies $\xi_{1}, \xi_{2}$.

The statements concerning $S\left(\bar{\psi}_{\sigma}\right)$ and the absolute continuity of $\bar{\psi}_{\sigma}$ are obvious consequences of Theorem 19. For the density $\bar{D}_{\sigma}(x)$ we find $\bar{D}_{\sigma}(x)$ $=|x|^{-2} \Delta_{\sigma}(\log x)$ where

$$
\Delta_{\sigma}(x)=\sum_{\mu=-\infty}^{\infty} D_{\sigma}(x+2 \pi i \mu) .
$$


From Theorem 19 we conclude that $\Delta_{\sigma}(x)$ is a continuous function of $x$ possessing continuous partial derivatives of arbitrarily high order. Also, if $\lambda>0$ then $\Delta_{\sigma}(x)=O\left(e^{-\lambda \xi_{1}}\right)$ as $\left|\xi_{1}\right| \rightarrow \infty$ and every partial derivative of $\Delta_{\sigma}(x)$ also is $=O\left(e^{-\lambda \xi_{1}^{2}}\right)$ as $\left|\xi_{1}\right| \rightarrow \infty$. This proves the continuity of $\bar{D}_{\sigma}(x)$ and the existence and continuity of its partial derivatives for $x \neq 0$. Furthermore,

$$
\bar{D}_{\sigma}(x)=O\left(|x|^{-2} e^{-\lambda(\log |x|) 2}\right)
$$

as $|x| \rightarrow \infty$ or $|x| \rightarrow 0$, while every partial derivative of $\bar{D}_{\sigma}(x)$ of order $p$ is $=O\left(|x|^{-2-p} e^{\left.-\lambda(\log |x|)^{2}\right)}\right.$ as $|x| \rightarrow \infty$ or $|x| \rightarrow 0$. Since $\lambda>0$ is arbitrary the factors $|x|^{-2}$ and $|x|^{-2-p}$ may be omitted. These appraisals imply the continuity of $\bar{D}_{\sigma}(x)$ and its partial derivatives at $x=0$ also, at which point all these functions vanish. That $\bar{D}_{\sigma}(x)>0$ for $x \neq 0$ if $\frac{1}{2}<\sigma \leqq 1$ is clear from Theorem 19.

Placing $\psi_{\sigma}^{\prime}=\phi_{1, \sigma} * \phi_{3, \sigma} * \cdots$ and $\psi_{\sigma}^{\prime \prime}=\phi_{2, \sigma} * \phi_{4, \sigma} * \cdots$ we have $\Lambda\left(y ; \psi_{\sigma}\right)$ $=\Lambda\left(y ; \psi_{\sigma}^{\prime}\right) \Lambda\left(y ; \psi_{\sigma}^{\prime \prime}\right)$, hence

$$
\frac{\partial}{\partial \eta_{2}} \Lambda\left(y ; \psi_{\sigma}\right)=\Lambda\left(y ; \psi_{\sigma}^{\prime}\right) \frac{\partial}{\partial \eta_{2}} \Lambda\left(y ; \psi_{\sigma}^{\prime \prime}\right)+\Lambda\left(y ; \psi_{\sigma}^{\prime \prime}\right) \frac{\partial}{\partial \eta_{2}} \Lambda\left(y ; \psi_{\sigma}^{\prime}\right),
$$

the existence and continuity of the partial derivatives being implied by the finiteness of $M_{1}\left(\psi_{\sigma}\right), M_{1}\left(\psi_{\sigma}^{\prime}\right), M_{1}\left(\psi_{\sigma}^{\prime \prime}\right)$, which is assured by Theorem 16. The finiteness of these numbers also implies the boundedness of the partial derivatives. Hence

$$
\frac{\partial}{\partial \eta_{2}} \Lambda\left(y ; \psi_{\sigma}\right)=O(|y|-q)
$$

for every $q$. Since by the inversion formula we have for every $n$

$$
\sum_{\mu=-n}^{n} D_{\sigma}(x+2 \pi i \mu)=(2 \pi)^{-2} \int_{R_{y}} e^{-i x y} \Lambda\left(y ; \psi_{\sigma}\right) \frac{\sin (2 n+1) \pi \eta_{2}}{\sin \pi \eta_{2}} m\left(d R_{y}\right),
$$

where $y=\eta_{1}+i \eta_{2}$, it is clear that

$$
\Delta_{\sigma}(x)=(2 \pi)^{-2} \int_{-\infty}^{\infty} \sum_{\nu=-\infty}^{\infty} e^{-i x\left(\eta_{1}+i \nu\right)} \Lambda\left(\eta_{1}+i \nu ; \psi_{\sigma}\right) d \eta_{1} .
$$

From this representation of $\Delta_{\sigma}(x)$ as a combined Fourier series and Fourier integral follows, in particular, by an argument exactly like the one applied in $\S 3$, that $\Delta_{\sigma}(x)$ is an entire function of the two variables $\xi_{1}, \xi_{2}$ if $\frac{1}{2}<\sigma<1$. This completes the proof of Theorem 21.

The last statement of Theorem 21 implies that if $\frac{1}{2}<\sigma<1$, then $\bar{D}_{\sigma}(x)$ is regular analytic at every point $x \neq 0$ of the real plane $R_{x}$; the point $x=0$ is 
actually an exception since at this point $\bar{D}_{\sigma}(x)$ and all its partial derivatives vanish.

By means of (10.1) it is easy to discuss $\bar{D}_{\sigma}(x)$ as a function of $\sigma$. We give only one result in this direction, which corresponds to Theorem 20:

THEOREM 22. The function $\bar{D}_{\sigma}(x)$ multiplied by $|x|^{2}$ and each of its partial derivatives of order $p$ multiplied by $|x|^{2+p}$ tend uniformly to zero as $\sigma \rightarrow \frac{1}{2}$.

The theorem is equivalent to the statement that $\Delta_{\sigma}(x)$ and each of its partial derivatives tend uniformly to zero as $\sigma \rightarrow \frac{1}{2}$, which follows from (10.1) by the argument used in the proof of Theorem 20.

We do not know whether Theorem 22 holds if the factors $|x|^{2}$ and $|x|^{2+p}$ are omitted; we do not even know whether $\bar{\psi}_{\sigma}(E) \rightarrow 0$ as $\sigma \rightarrow \frac{1}{2}$ for any bounded set $E$.

\section{Asymptotic DISTRIBUTION FUNCTIONS}

Let $G$ be an abstract space with $t$ as variable point and in $G$ let there be defined a measure $m$ such that the system of sets $A$ for which $m(A)$ is defined is a Borel field and $m$ is non-negative and completely additive; we suppose that $G$ belongs to the system and that $m(G)=\infty$. The sets $A$ for which $m(A)$ is defined are called measurable sets. Lebesgue integrals with respect to $m$ will be denoted by

$$
\int_{A} f(t) m(d G) .
$$

We suppose that certain sequences $A_{1}, A_{2}, \cdots$ consisting of measurable sets of positive finite measure such that $m\left(A_{n}\right) \rightarrow \infty$ have been selected and call these sequences admissible sequences. A real or complex function $f(t)$ which is measurable in $G$ is said to have the mean value $M(f)=M(f(t))$ if

$$
M(f)=\lim _{n \rightarrow \infty} \frac{1}{m\left(A_{n}\right)} \int_{A_{n}} f(t) m(d G)
$$

for each admissible sequence. A measurable set $A$ in $G$ is said to have the relative measure $\rho(A)$ if

$$
\rho(A)=\lim _{n \rightarrow \infty} \frac{m\left(A A_{n}\right)}{m\left(A_{n}\right)}
$$

for each admissible sequence. If $f(t) \geqq 0$ is measurable in $G$ its upper mean value $\bar{M}(f)=\bar{M}(f(t))$ is the least upper bound of

$$
\limsup _{n \rightarrow \infty} \frac{1}{m\left(A_{n}\right)} \int_{A_{n}} f(t) m(d G)
$$


for all admissible sequences. Similarly, if $A$ is a measurable set in $G$ its upper relative measure $\bar{\rho}(A)$ is the least upper bound of

$$
\limsup _{n \rightarrow \infty} \frac{m\left(A A_{n}\right)}{m\left(A_{n}\right)}
$$

for all admissible sequences. These notions depend, of course, on the definition of admissible sequences, which is supposed to be fixed once for all. It is clear when for a class of functions or sets the mean values or relative measures shall be said to exist uniformly for all functions or sets of the class.

Let $x(t)$ be a measurable vector function with $k$ components defined in $G$. Then if $E$ is a Borel set in $R_{x}$, the set $A_{E}$ of those points $t$ in $G$ for which $x(t)$ belongs to $E$ is measurable. We say that $x(t)$ has an asymptotic distribution function if there exists in $R_{x}$ a distribution function $\phi$ such that for each continuity set $E$ of $\phi$ the relative measure $\rho\left(A_{E}\right)$ exists and is $=\phi(E)$. It is clear that there exists at most one such distribution function $\phi$. The restriction imposed on $E$ that it should be a continuity set for $\phi$ is essential as will be seen from later examples. Another form of the definition is the following: For a measurable set $A$ in $G$ of positive finite measure, let $\phi_{A}$ denote the distribution function defined by

$$
\phi_{A}(E)=\frac{m\left(A_{E} A\right)}{m(A)} ;
$$

then $x(t)$ possesses an asymptotic distribution function $\phi$ if and only if $\phi_{A_{n}} \rightarrow \phi$ for any admissible sequence.

THEOREM 23. The vector function $x(t)$ possesses an asymptotic distribution function $\phi$ if and only if the mean value $M\left(e^{i x(t) y}\right)$ exists and

$$
M\left(e^{i x(t) y}\right)=\lim _{n \rightarrow \infty} \frac{1}{m\left(A_{n}\right)} \int_{A_{n}} e^{i x(t) y} m(d G)
$$

holds uniformly in every sphere $|y| \leqq a$ in $R_{y}$ for any admissible sequence, and we have then $\Lambda(y ; \phi)=M\left(e^{i x(t)} y\right)$.

For any measurable set $A$ in $G$ of positive finite measure we have

$$
\Lambda\left(y ; \phi_{A}\right)=\frac{1}{m(A)} \int_{A} e^{i x(t)} y_{m}(d G) ;
$$

the theorem follows therefore from $\$ 3$.

A sequence of vector functions $x_{1}(t), x_{2}(t), \cdots$ is said to be convergent in relative measure to the limit function $x(t)$ if $\bar{\rho}\left(\left|x(t)-x_{n}(t)\right|>\epsilon\right) \rightarrow 0$ as $n \rightarrow \infty$ for every fixed $\epsilon>0$. From $\$ 3$ and Theorem 23 follows immediately 
THEOREM 24. If $x_{1}(t), x_{2}(t), \cdots$ is a sequence of measurable vector functions which converges in relative measure to the vector function $x(t)$ and if every $x_{n}(t)$ has an asymptotic distribution function $\phi_{n}$, then $x(t)$ also possesses an asymptotic distribution function $\phi$ and $\phi_{n} \rightarrow \phi$.

The existence of a distribution function $\phi$ of $x(t)$ implies immediately the existence of the mean value $M(F(x(t)))$ for any bounded continuous function $F(x)$ in $R_{x}$ and also gives the formula

$$
M(F(x(t)))=\int_{R_{x}} F(x) \phi\left(d R_{x}\right) .
$$

Hence we conclude that if the mean value $M(F(x(t)))$ exists for the function $F(x)=e^{i x y}$ where $y$ is arbitrary, and if the limit relation (11.1) holds uniformly in every sphere $|y| \leqq a$ for any admissible sequence, then $M(F(x(t)))$ exists for any bounded continuous function $F(x)$ in $R_{x}$. One may start, of course, with other systems than the system of the functions $F(x)=e^{i x y}$ in order to obtain conditions for the existence of $\phi$. In this direction we mention the theorem that if $|x(t)|$ is bounded then the existence of $M(F(x(t)))$ for any $F(x)=\xi_{1} q_{1} \cdots \xi_{k} q_{k}$ is necessary and sufficient for the existence of $\phi$. This condition is equivalent to the one that every moment $\mu_{q_{1}}, \ldots, q_{k}\left(\phi_{A}\right)$ approaches for any admissible sequence a limit which is then $\mu_{q_{1}, \cdots, q_{k}}(\phi)$. This method still applies when $|x(t)|$ is not bounded but such that the limits of the moments belong to a determined moment problem (cf. Wintner [49], [54] and Fréchet and Shohat [25]). Hence the moment method applies only under restrictive conditions regarding $x(t)$ while the method of the Fourier transform applies whenever $x(t)$ has an asymptotic distribution function.

It is sometimes of interest to establish the existence of the mean value $M(F(x(t)))$ also for unbounded functions $F(x)$. In this direction we have the theorem (cf. Bohr and Jessen [21]) that if $x(t)$ has an asymptotic distribution function $\phi$ and if for some continuous function $H(x) \geqq 0$ the upper mean value $\bar{M}(H(x(t)))$ is finite, then $M(F(x(t)))$ exists for any continuous function $F(x)$ satisfying the condition $F(x)=o(H(x))$ as $|x| \rightarrow \infty$ and (11.2) is valid.

\section{ASYMPtOTIC DISTRIBUTION FUNCTIONS OF ALMOST PERIODIC FUNCTIONS}

Let $G$ be the real axis $-\infty<t<\infty$ and $m$ the Lebesgue measure on it. Two cases will be considered; in the first case an admissible sequence is an arbitrary sequence of intervals $\left(a_{n}, b_{n}\right)$ where $b_{n}-a_{n} \rightarrow \infty$; in the second case we allow only sequences $\left(0, b_{n}\right)$ where $b_{n} \rightarrow \infty$ and sequences $\left(a_{n}, 0\right)$ where $a_{n} \rightarrow-\infty$. We shall refer to the two cases as the unrestricted case and the restricted case, but we shall not distinguish the two cases by the use of different 
notations for mean values, relative measures, etc. The existence of an asymptotic distribution function in the unrestricted case implies of course the existence in the restricted case also.

A vector function $x(t)$ is called almost periodic if each of its $k$ components is almost periodic. Our notations will be those used by Besicovitch [1] so that in particular a u.a.p. function means a function almost periodic in the original sense of Bohr.

THEOREM 25. Any u.a.p. vector function $x(t)$ possesses an asymptotic distribution function in the unrestricted case.

The functions $e^{i x(t) y}$ form for $|y| \leqq a$ a majorisable class of $u . a . p$. functions; consequently the mean value

$$
M\left(e^{i x(t) y}\right)=\lim _{n \rightarrow \infty} \frac{1}{b_{n}-a_{n}} \int_{a_{n}}^{b_{n}} e^{i x(t) y} d t
$$

exists uniformly for $|y| \leqq a$ for any admissible sequence.

THEOREM 26. The spectrum $S(\phi)$ of the asymptotic distribution function $\phi$ of a u.a.p. vector function $x(t)$ is the closure of the range of $x(t)$.

Since the range of $x(t)$ is defined as the set of those points $x_{0}$ of $R_{x}$ for which $x_{0}=x\left(t_{0}\right)$ holds for some $t_{0}$ it is clear that any point of $S(\phi)$ belongs to the closure of the range of $x(t)$. Conversely, if $x_{0}=x\left(t_{0}\right)$ for some $t_{0}$ then $x_{0}$ belongs to $S(\phi)$, which means that $\bar{\rho}\left(\left|x(t)-x\left(t_{0}\right)\right|<\epsilon\right)>0$ for every $\epsilon>0$. This is an easy consequence of the uniform continuity of $x(t)$ and the fact that $\left|x(t)-x\left(t_{0}\right)\right|<\epsilon / 2$ for a relatively dense set of values $t$.

A measurable vector function $x(t)$ is $W a . p$. if and only if there exists a sequence $x_{1}(t), x_{2}(t), \cdots$ of $u . a . p$. vector functions such that

$$
\bar{M}\left(\left|x(t)-x_{n}(t)\right|\right) \rightarrow 0 \text { as } n \rightarrow \infty
$$

where the upper mean value belongs to the unrestricted case. A measurable vector function $x(t)$ is $B a . p$. if and only if the same holds with the sole difference that the upper mean value belongs to the restricted case.

ThEOREM 27. Any $W$ a.p. vector function $x(t)$ possesses an asymptotic distribution function in the unrestricted case. Any $B$ a.p. vector function possesses an asymptotic distribution function in the restricted case.

Let $x(t)$ be $W a . p . ;$ from (12.1) and the inequality

$$
\bar{\rho}\left(\left|x(t)-x_{n}(t)\right|>\epsilon\right) \leqq \epsilon^{-1} \bar{M}\left(\left|x(t)-x_{n}(t)\right|\right)
$$

we deduce

$$
\bar{\rho}\left(\left|x(t)-x_{n}(t)\right|>\epsilon\right) \rightarrow 0 \text { as } n \rightarrow \infty
$$


where the upper relative measure belongs to the unrestricted case. Hence the result follows from Theorem 24. If $x(t)$ is $B a . p$. the proof is the same with the sole difference that the upper relative measure belongs to the restricted case.

Let $x(t)$ be an almost periodic vector function (of one of the types considered) and $\phi$ its asymptotic distribution function; let $E$ be a Borel set in $R_{x}$; then if $A_{E}$ denotes the set of those points $t$ for which $x(t)$ belongs to $E$ we know that $\rho\left(A_{E}\right)$ exists and is $=\phi(E)$ for any continuity set $E$ of $\phi$, the relative measure belonging to the unrestricted case if $x(t)$ is $W$ a.p. and to the restricted case if $x(t)$ is $B a . p$. If $E$ is not a continuity set of $\phi$ the relative measure $\rho\left(A_{E}\right)$ need not exist and even when it exists it need not be $=\phi(E)$. A simple example (for $k=1$ ) of the first behavior was given by Bohr [17] who constructed a u.a.p. function $x(t)$ for which $\rho\left(A_{E}\right)$ does not exist (not even in the restricted case) for a certain interval $E$. An example of the second behavior (for $k=2$ ) is the function $x(t)=\log \zeta(\sigma+i t)$ where $\sigma>1$ is fixed; this function is $u . a . p$. and its asymptotic distribution function is absolutely continuous $(\$ 14)$; on the other hand, the range of $x(t)$ is a null-set; hence if we take $E$ to be the range of $x(t)$, we have $\rho\left(A_{E}\right)=1$ and $\phi(E)=0$.

Let now $E$ be a continuity set of $\phi$ and let $f(t)$ denote the function which is 1 or 0 according as $x(t)$ does or does not belong to $E$. Then we know that $M(f(t))$ exists and is $=\phi(E)$. It is natural to ask if $f(t)$ is also almost periodic (in some sense). We shall prove that $f(t)$ is $W a . p$. if $x(t)$ is $W a . p$. and $B a . p$. if $x(t)$ is $B$ a.p. We prove this as follows: The fact that $E$ is a continuity set of $\phi$ makes it possible, corresponding to any given $\epsilon>0$, to find two continuous functions $F(x)$ and $G(x)$ in $R_{x}$ such that $0 \leqq F(x) \leqq G(x) \leqq 1$ for all $x$, $F(x)=0$ when $x$ does not belong to $E, G(x)=1$ when $x$ belongs to $E$, and finally

$$
\int_{R_{x}}(G(x)-F(x)) \phi\left(d R_{x}\right)<\epsilon .
$$

This implies $F(x(t)) \leqq f(t) \leqq G(x(t))$ and also

$$
M(G(x(t))-F(x(t)))<\epsilon
$$

where the mean value belongs to the unrestricted or to the restricted case according as $x(t)$ is $W a . p$. or $B a . p$. Now $F(x(t))$ and $G(x(t))$ are $W a . p$. if $x(t)$ is $W a . p$. and $B$ a.p. if $x(t)$ is $B a . p$. This leads to the desired conclusion.

If $x(t)$ is $u . a . p$. we find that $f(t)$ is $W a . p$. and we cannot say more than this; in particular we cannot say that $f(t)$ is $S$ a.p. This is shown (for $k=1$ ) by the following example: Let $x_{1}(t)$ denote the periodic function with period 4 which is $=0$ for $|x| \leqq 1$ and $=|x|-1$ for $1 \leqq|x| \leqq 2$ and let $x_{n}(t)$ denote the function $x_{n}(t)=2^{-n} x_{1}\left(2^{-n} t\right)$. Let $x(t)$ be the $u . a . p$. function defined by the 
uniformly convergent series $x(t)=x_{1}(t)+x_{2}(t)+\cdots$. Then the set $x=0$ is a continuity set of the asymptotic distribution function $\phi$ of $x(t)$. The corresponding function $f(t)$ is $=1$ for $|x| \leqq 1$ and $=0$ elsewhere; hence $f(t)$ is not $S$ a.p.

The methods of this section may be extended to functions of two or more variables; for a result in this direction cf. Wintner [58].

\section{INDEPENDENT MODULI}

By the modul of a $B$ a.p. vector function is understood the smallest modul containing the Fourier exponents of each of the $k$ components $\xi_{1}(t), \cdots, \xi_{k}(t)$ of $x(t)$. The $B$ a.p. vector functions $x_{1}(t), x_{2}(t), \cdots$ are said to have independent moduli if a finite sum $\alpha_{1}+\cdots+\alpha_{n}$, where $\alpha_{\nu}$ belongs to the modul of $x_{\nu}(t)$, is equal to zero only when all $\alpha_{\nu}=0$.

THEOREM 28. If $x_{1}(t), \cdots, x_{n}(t)$ are $B$ a.p. vector functions with independent moduli then the asymptotic distribution function $\phi$ of $x(t)=x_{1}(t)+\cdots+x_{n}(t)$ is $\phi_{1} * \cdots * \phi_{n}$ where $\phi_{1}, \cdots, \phi_{n}$ are the asymptotic distribution functions of $x_{1}(t), \cdots, x_{n}(t)$ respectively.

The statement is that $\Lambda(y ; \phi)=\Lambda\left(y ; \phi_{1}\right) \cdots \Lambda\left(y ; \phi_{n}\right)$ which may be written according to Theorem 23 in the form

$$
M\left(e^{i\left(x_{1}(t)+\cdots+x_{n}(t)\right) y}\right)=M\left(e^{i x_{1}(t) y}\right) \cdots M\left(e^{i x_{n}(t) y}\right)
$$

where the mean values belong to the restricted case. In virtue of the approximation theorem for $B$ a.p. functions it is enough to verify the last relation in the case where all components of $x_{1}(t), \cdots, x_{n}(t)$ are exponential polynomials in which case it follows by a direct calculation.

With regard to this argument cf. Wintner [53-55], Bochner and Jessen [3]; this is the point where the explicit use of the theory of diophantine approximations is avoided as pointed out in $\$ 1$.

The conditions of Theorem 28 are in particular satisfied if $x_{1}(t), \cdots, x_{n}(t)$ are periodic vector functions with periods $2 \pi / \lambda_{1}, \cdots, 2 \pi / \lambda_{n}$ where $\lambda_{1}, \cdots, \lambda_{n}$ are linearly independent.

For $k=2$ we have as a simple application

TheOREM 29. Let $r_{1}, r_{2}, \cdots$ be positive, $\lambda_{1}, \lambda_{2}, \cdots$ linearly independent, and $\delta_{1}, \delta_{2}, \cdots$ real. Then the asymptotic distribution function of $s_{n}(t)$ $=r_{1} e^{i\left(\lambda_{1} t+\delta_{1}\right)}+\cdots+r_{n} e^{i\left(\lambda_{n} t+\delta_{n}\right)}$ is the distribution function $\psi_{n}=\phi_{1} * \cdots * \phi_{n}$ described in Theorem 10. If $r_{1} e^{i\left(\lambda_{1} t+\delta_{1}\right)}+r_{2} e^{i\left(\lambda_{2} t+\delta_{2}\right)}+\cdots$ is the Fourier series of a $B$ a.p. function $s(t)$, then $r_{1}^{2}+r_{2}^{2}+\cdots$ is convergent and the asymptotic distribution function of $s(t)$ is the distribution function $\psi=\phi_{1} * \phi_{2} * \cdots$ described in Theorem 10. Finally, if $s(t)$ is bounded then $r_{1}+r_{2}+\cdots$ converges. 
The first part of the theorem is an immediate consequence of Theorem 28 since the asymptotic distribution function of a pure oscillation $r e^{i(\lambda t+\delta)}$ is the circular equidistribution on $|x|=r$. The second part of the theorem is clear from the relation

$$
M\left(\left|s(t)-s_{n}(t)\right|\right) \rightarrow 0 \text { as } n \rightarrow \infty
$$

where the mean value belongs to the restricted case; this relation is a consequence of the approximation theorem for $B$ a.p. functions. Use is made of the linear independence of the exponents. Finally, the last part of the theorem follows from Theorem 10 since the boundedness of $s(t)$ implies the boundedness of $S(\psi)$. In this last part of the theorem is contained the classical theorem of Bohr that the Fourier series of a u.a.p. function with linearly independent exponents is absolutely convergent; it is interesting that the original proof of Bohr [16], without using the notion of a distribution function, was built precisely on the same ideas as the present proof.

It is interesting that the smoothness of the asymptotic distribution function $\psi$ of $s(t)$ established by Theorem 29 does not imply any smoothness for $s(t)$ itself. In fact (cf. Wintner [56]), if $r_{n}=a^{n}, \lambda_{n}=b^{n}$ and $\delta_{n}=0$ where $0<a<1, a b \geqq 1$ and $b$ is a transcendental number, then $s(t)$ is u.a.p. with linearly independent exponents but is nowhere differentiable.

\section{The RIEMANN zeta function}

We now consider the Riemann zeta function $\zeta(s)=\zeta(\sigma+i t)$. In the halfplane $\sigma>1$ we have

$$
\zeta(s)=\prod_{n=1}^{\infty}\left(1-p_{n}^{-s}\right)^{-1}
$$

where $p_{1}, p_{2}, \cdots$ denote the primes $2,3, \cdots$; in particular, $\zeta(s) \neq 0$ for $\sigma>1$. We write

$$
\zeta_{n}(s)=\prod_{v=1}^{n}\left(1-p_{\nu}^{-s}\right)^{-1}
$$

$\zeta_{n}(s)$ is regular and $\neq 0$ for $\sigma>0$. By $\log \zeta(s)$ and $\log \zeta_{n}(s)$ we denote the functions

and

$$
\log \zeta(s)=\sum_{n=1}^{\infty}-\log \left(1-p_{n}^{-s}\right)
$$

$$
\log \zeta_{n}(s)=\sum_{\nu=1}^{n}-\log \left(1-p_{\nu}^{-s}\right)
$$


where in each term on the right $-\log (1-z)=z+\frac{1}{2} z^{2}+\cdots$. The function $\log \zeta(s)$ is regular for $\sigma>1$ and $\log \zeta_{n}(s)$ for $\sigma>0$. By $H$ we denote the domain obtained from the half-plane $\sigma>\frac{1}{2}$ by leaving out the segment $\frac{1}{2}<\sigma \leqq 1, t=0$, and all segments $\frac{1}{2}<\sigma \leqq \sigma_{0}, t=t_{0}$, where $\sigma_{0}+i t_{0}$ denote the zeros (if any) of $\zeta(s)$ in $\sigma>\frac{1}{2}$; by $\log \zeta(s)$ for $\sigma>\frac{1}{2}$ we understand the analytic continuation of $\log \zeta(s)$ in $H$. For any fixed $\sigma>1$ the functions $\zeta(\sigma+i t)$ and $\log \zeta(\sigma+i t)$ are both u.a.p.; similarly, $\zeta_{n}(\sigma+i t)$ and $\log \zeta_{n}(\sigma+i t)$ are u.a.p. for any fixed $\sigma>0$ and tend uniformly to $\zeta(\sigma+i t)$ and $\log \zeta(\sigma+i t)$ if $\sigma>1$. For any fixed $\sigma>\frac{1}{2}$ the function $\zeta(\sigma+i t)$ is $B^{2} a . p$. and

$$
M\left(\left|\zeta(\sigma+i t)-\zeta_{n}(\sigma+i t)\right|^{2}\right) \rightarrow 0 \text { as } n \rightarrow \infty,
$$

where the mean value belongs to the restricted case. This follows, e.g., from a result of Besicovitch [1], pp. 163-169, but is in the main of an older date. In the case $\sigma=1$ it is necessary in all integrations to leave out a vicinity of the pole $t=0$. Finally, it was proved by Bohr [12] that if $\sigma>\frac{1}{2}$ is fixed then

$$
\bar{\rho}\left(\left|\log \zeta(\sigma+i t)-\log \zeta_{n}(\sigma+i t)\right|>\epsilon\right) \rightarrow 0 \text { as } n \rightarrow \infty
$$

for any $\epsilon>0$; the upper relative measure belongs to the restricted case.

Using our previous results we can now prove very easily

THEOREM 30. The function $\log \zeta(\sigma+i t)$ possesses an asymptotic distribution function in the unrestricted case if $\sigma>1$ and in the restricted case if $\frac{1}{2}<\sigma \leqq 1$. This asymptotic distribution function is the distribution function $\psi_{\sigma}=\phi_{1, \sigma}$ ${ }^{*} \phi_{2, \sigma} * \cdots$ described in Theorems 19 and 20. The closure of the range of $\log \zeta(\sigma+i t)$ is $S\left(\psi_{\sigma}\right)$.

The first part of the theorem follows from Theorems 25 and 24. In order to prove the second part of the theorem we first observe that if $S$ denotes the curve $x=x(\theta)=-\log \left(1-r e^{2 \pi i \theta}\right)$ where $0<r<1$, and if $\phi$ is the distribution function in $R_{x}$ determined by this parametric representation of $S$, then $\phi$ is also for an arbitrary $\lambda \neq 0$ the asymptotic distribution function of the function $-\log \left(1-r e^{i \lambda t}\right)$. Now if $\sigma>0$ then

$$
\log \zeta_{n}(\sigma+i t)=\sum_{\nu=1}^{n}-\log \left(1-p_{\nu}^{-\sigma} e^{-i \log p_{\nu} t}\right)
$$

since the numbers $\log p_{1}, \cdots, \log p_{n}$ are linearly independent it follows from Theorem 28 that $\log \zeta_{n}(\sigma+i t)$ has the asymptotic distribution function $\phi_{1, \sigma} * \cdots * \phi_{n, \sigma}$. The second part of the theorem follows then by Theorem 24 . Finally, the last part of the theorem is a consequence of Theorem 26 if $\sigma>1$ and is clear if $\frac{1}{2}<\sigma \leqq 1$ since $S\left(\psi_{\sigma}\right)$ is then the whole $R_{x}$.

For $\zeta(s)$ itself we have a corresponding result: 
THEOREM 31. The function $\zeta(\sigma+i t)$ possesses an asymptotic distribution function in the unrestricted case if $\sigma>1$ and in the restricted case if $\frac{1}{2}<\sigma \leqq 1$. This asympitotic distribution function is the distribution function $\bar{\psi}_{\sigma}$ described in Theorems 21 and 22. The closure of the range of $\zeta(\sigma+i t)$ is $S\left(\bar{\psi}_{\sigma}\right)$.

The first part of the theorem follows from Theorems 25 and 27; the second part is an immediate consequence of the definition of $\bar{\psi}_{\sigma}$; finally, the last part of the theorem is a consequence of Theorem 26 if $\sigma>1$ and is clear if $\frac{1}{2}<\sigma \leqq 1$ since $S\left(\bar{\psi}_{\sigma}\right)$ is then the whole $R_{x}$.

It is clear that the relations

$$
\bar{D}_{\sigma}(0)=0 ; \quad \bar{D}_{\sigma}(x)>0, \quad x \neq 0 \quad\left(\frac{1}{2}<\sigma \leqq 1\right)
$$

may be interpreted as an illustration to the Riemann hypothesis.

\section{Measure AND INTEGRATION IN PRODUCT SPACES}

We obtain further results concerning infinite convolutions by using the theory of measure and integration in product spaces of an infinite number of spaces. In the present section we collect some of the results of this theory. These results are proved for a special case in Jessen [35] where references to the literature are to be found. The proofs for the general case will be given by Jessen in a forthcoming paper.

Let $Q$ be an abstract space with $t$ as variable point and let there be defined in $Q$ a measure $m$ such that the system of sets $A$ for which $m(A)$ is defined is a Borel field and $m$ is non-negative and completely additive. We suppose that $Q$ itself belongs to the field and that $m(Q)=1$. The sets $A$ for which $m(A)$ is defined are called measurable sets; Lebesgue integrals with respect to $m$ are denoted by

$$
\int_{A} f(t) m(d Q) .
$$

It is well known that if $q_{1}$ and $q_{2}$ are two spaces of the type described before, with $\tau_{1}$ and $\tau_{2}$ as variable points and with $\mu_{1}$ and $\mu_{2}$ as measures, then these measures generate in the product space $Q=\left(q_{1}, q_{2}\right)$ with the variable point $t=\left(\tau_{1}, \tau_{2}\right)$ a measure $m=\left(\mu_{1}, \mu_{2}\right)$ in the following way. The system of sets in $Q$ for which $m$ is defined is the smallest Borel field containing all sets $A=\left(a_{1}, a_{2}\right)$ of $Q$ where $a_{1}$ and $a_{2}$ are measurable sets in $q_{1}$ and $q_{2}$ respectively and $m$ is characterized by the property that if $A=\left(a_{1}, a_{2}\right)$ then $m(A)$ $=\mu_{1}\left(a_{1}\right) \mu_{2}\left(a_{2}\right)$. For integrals with respect to $m$ we have Fubini's theorem

$$
\int_{Q} f(t) m(d Q)=\int_{q} \mu_{2}\left(d q_{2}\right) \int_{q} f\left(\tau_{1}, \tau_{2}\right) \mu_{1}\left(d q_{1}\right) .
$$


Let $q_{1}, q_{2}, \cdots$ be a finite or infinite sequence of abstract spaces of the type described above, with $\tau_{1}, \tau_{2}, \cdots$ as variable points and $\mu_{1}, \mu_{2}, \cdots$ as measures. Let $Q$ denote the space $Q=\left(q_{1}, q_{2}, \cdots\right)$ where $t=\left(\tau_{1}, \tau_{2}, \cdots\right)$ is the variable point; then the measures $\mu_{1}, \mu_{2}, \cdots$ generate a measure $m=\left(\mu_{1}, \mu_{2}, \cdots\right)$ in $Q$ in the following way. The system of sets in $Q$ for which $m$ is defined is the smallest Borel field containing all sets $A=\left(a_{1}, a_{2}, \cdots\right)$, where $a_{1}, a_{2}, \cdots$ are measurable sets in $q_{1}, q_{2}, \cdots$ respectively and $m$ is characterized by the property that for sets of this type we have $m(A)$ $=\mu_{1}\left(a_{1}\right) \mu_{2}\left(a_{2}\right) \cdots$.

Suppose now that the sequence $q_{1}, q_{2}, \ldots$ is infinite; then we may for every $n$ consider the space $Q_{n}=\left(q_{1}, \cdots, q_{n}\right)$ with $t_{n}=\left(\tau_{1}, \cdots, \tau_{n}\right)$ as variable point and with $m_{n}=\left(\mu_{1}, \cdots, \mu_{n}\right)$ as measure and the space $Q_{n, \omega}=\left(q_{n+1}\right.$, $\left.q_{n+2}, \cdots\right)$ with $t_{n, \omega}=\left(\tau_{n+1}, \tau_{n+2}, \cdots\right)$ as variable point and $m_{n, \omega}=\left(\mu_{n+1}\right.$, $\left.\mu_{n+2}, \cdots\right)$ as measure. Then $Q=\left(q_{1}, q_{2}, \cdots\right)=\left(Q_{n}, Q_{n, \omega}\right), t=\left(\tau_{1}, \tau_{2}, \cdots\right)$ $=\left(t_{n}, t_{n, \omega}\right)$ and it is easily seen that $m=\left(\mu_{1}, \mu_{2}, \cdots\right)=\left(m_{n}, m_{n, \omega}\right)$. Hence if $f(t)$ is integrable in $Q$ we have

$$
\begin{aligned}
\int_{Q} f(t) m(d Q) & =\int_{Q_{n}} m_{n}\left(d Q_{n}\right) \int_{Q_{n, \omega}} f\left(t_{n}, t_{n, \omega}\right) m_{n, \omega}\left(d Q_{n, \omega}\right) \\
& =\int_{Q_{n, \omega}} m_{n, \omega}\left(d Q_{n, \omega}\right) \int_{Q_{n}} f\left(t_{n}, t_{n, \omega}\right) m_{n}\left(d Q_{n}\right)
\end{aligned}
$$

for every $n$. We have now the following theorems:

TheOREM A. Let $f(t)$ be integrable in $Q$ and let $f_{n}(t)$ denote the function

$$
f_{n}(t)=\int_{Q_{n, \omega}} f\left(t_{n}, t_{n, \omega}\right) m_{n, \omega}\left(d Q_{n, \omega}\right)
$$

so that $f_{n}(t)$ depends only on $t_{n}$. Then $f_{n}(t) \rightarrow f(t)$ almost everywhere in $Q$ as $n \rightarrow \infty$.

THEOREM B. Let $A$ be a measurable set in $Q$ with the property that two points $t^{\prime}=\left(\tau_{1}^{\prime}, \tau_{2}^{\prime}, \cdots\right)$ and $t^{\prime \prime}=\left(\tau_{1}^{\prime \prime}, \tau_{2}^{\prime \prime}, \ldots\right)$ such that $\tau_{n}^{\prime}=\tau_{n}^{\prime \prime}$ when $n>n_{0}$ $=n_{0}\left(t^{\prime}, t^{\prime \prime}\right)$ always either both belong to $A$ or both do not belong to $A$. Then $m(A)$ is either 0 or 1 .

THEOREM C. Let $f(t)$ be integrable in $Q$ and let $f_{n, \omega}(t)$ denote the function

$$
f_{n, \omega}(t)=\int_{Q_{n}} f\left(t_{n}, t_{n, \omega}\right) m_{n}\left(d Q_{n}\right)
$$

so that $f_{n, \omega}(t)$ depends only on $t_{n, \omega}$. Then $f_{n, \omega}(t) \rightarrow I$ almost everywhere in $Q$ as $n \rightarrow \infty$ where $I$ is the constant

$$
I=\int_{Q} f(t) m(d Q)
$$




\section{THE CONVERGENCE PROBLEM OF INFINITE CONVOLUTIONS}

Let $Q$ be an abstract space of the type considered at the beginning of $\$ 15$ and let $x(t)$ be a measurable vector function with $k$ components defined on $Q$. Then if $E$ is a Borel set in $R_{x}$ the set $A_{E}$ of those points $t$ for which $x(t)$ belongs to $E$ is a measurable set in $Q$. The distribution function $\phi$ in $R_{x}$ defined by $\phi(E)=m\left(A_{E}\right)$ will be called the distribution function of $x(t)$ in $Q$. For the Fourier transform of $\phi$ we have

$$
\Lambda(y ; \phi)=\int_{Q} e^{i x(t) y} m(d Q) .
$$

For later application we notice that any distribution function $\phi$ in $R_{x}$ is the distribution function of a measurable vector function $x(t)$ in a suitable abstract space $Q$. The simplest possibility is to choose $Q$ as $R_{x}$ itself, except for the change in the notation for the variable point, and to choose $\phi$ as measure in $Q$. Then $x(t)=t$ is a measurable vector function in $Q$ and $\phi$ is its distribution function.

Let $Q=\left(q_{1}, q_{2}\right)$ and let $x_{1}\left(\tau_{1}\right)$ and $x_{2}\left(\tau_{2}\right)$ be measurable vector functions with $k$ components in $q_{1}$ and $q_{2}$ and having the distribution functions $\phi_{1}$ and $\phi_{2}$. Then the distribution function $\phi$ of $x(t)=x_{1}\left(\tau_{1}\right)+x_{2}\left(\tau_{2}\right)$ in $Q$ is $\phi=\phi_{1} * \phi_{2}$. This follows readily from the definitions and is obvious also from (15.1) and (16.1) which imply $\Lambda(y ; \phi)=\Lambda\left(y ; \phi_{1}\right) \Lambda\left(y ; \phi_{2}\right)$. We shall now prove that a corresponding theorem holds for infinite convolutions also:

THEOREM 32. A necessary and sufficient condition for the convergence of the infinite convolution $\phi_{1} * \phi_{2} * \cdots$ is that if $q_{1}, q_{2}, \cdots$ are abstract spaces and $x_{1}\left(\tau_{1}\right), x_{2}\left(\tau_{2}\right), \cdots$ measurable functions in $q_{1}, q_{2}, \cdots$ having the distribution functions $\phi_{1}, \phi_{2}, \cdots$, then the series $x_{1}\left(\tau_{1}\right)+x_{2}\left(\tau_{2}\right)+\cdots$ is convergent almost everywhere in $Q=\left(q_{1}, q_{2}, \cdots\right)$. The distribution function of $s(t)=x_{1}\left(\tau_{1}\right)$ $+x_{2}\left(\tau_{2}\right)+\cdots$ is then $\psi=\phi_{1} * \phi_{2} * \cdots$.

The sum $s_{n}(t)=x_{1}\left(\tau_{1}\right)+\cdots+x_{n}\left(\tau_{n}\right)$ is for every $n$ a measurable vector function in $Q$ and its distribution function is $\psi_{n}=\phi_{1} * \cdots * \phi_{n}$. Similarly, the distribution function of $r_{n, p}(t)=x_{n+1}\left(\tau_{n+1}\right)+\cdots+x_{n+p}\left(\tau_{n+p}\right)$ is $\rho_{n, p}$ $=\phi_{n+1} * \cdots * \phi_{n+p}$. From Theorem 1 a necessary and sufficient condition for the convergence of $\phi_{1} * \phi_{2} * \cdots$ is that $\rho_{n, p} \rightarrow \chi_{0}$ as $n \rightarrow \infty$, which means that $r_{n, p}(t) \rightarrow 0$ in measure (en mesure) as $n \rightarrow \infty$. Hence the convergence in measure of the series $x_{1}\left(\tau_{1}\right)+x_{2}\left(\tau_{2}\right)+\cdots$ is necessary and sufficient for the convergence of $\phi_{1} * \phi_{2} * \cdots$, and it is also seen from (16.1) that the sum $s(t)=x_{1}\left(\tau_{1}\right)+x_{2}\left(\tau_{2}\right)+\cdots$ has then $\psi=\phi_{1} * \phi_{2} * \cdots$ as distribution function. Since convergence almost everywhere implies convergence in measure, it remains only to prove that for series of the type $x_{1}\left(\tau_{1}\right)+x_{2}\left(\tau_{2}\right)+\cdots$ con- 
vergence in measure implies convergence almost everywhere. If $s(t)=x_{1}\left(\tau_{1}\right)$ $+x_{2}\left(\tau_{2}\right)+\cdots$ is convergent in measure then

$$
e^{i s(t) y}=e^{i x_{1}\left(\tau_{1}\right) y} e^{i x_{2}\left(\tau_{2}\right) y} \ldots
$$

also holds in the sense of convergence in measure for every $y$ in $R_{y}$. For a fixed $y$ we write $f(t)=e^{i s(t)} y$; then $f(t)$ is measurable and bounded, hence integrable, so that we may apply Theorem A. We find

$$
f_{n}(t)=e^{i x_{1}\left(\tau_{1}\right) y} \cdots e^{i x_{n}\left(\tau_{n}\right) y} a_{n}
$$

where the constant $a_{n} \rightarrow 1$ as $n \rightarrow \infty$; hence

$$
e^{i s(t) y}=e^{i x_{1}\left(\tau_{1}\right) y} e^{i x_{2}\left(\tau_{2}\right) y} \ldots
$$

holds in the sense of convergence almost everywhere for every $y$, which shows that $x(t)=x_{1}\left(\tau_{1}\right)+x_{2}\left(\tau_{2}\right)+\cdots$ is convergent almost everywhere.

Since the set $A$ of points $t$ in $Q$ in which a series of the form $x_{1}\left(\tau_{1}\right)+x_{2}\left(\tau_{2}\right)$ $+\cdots$ is convergent satisfies the conditions of Theorem $B$, we have also

THEOREM 33. An infinite series $x_{1}\left(\tau_{1}\right)+x_{2}\left(\tau_{2}\right)+\cdots$ whose terms are measurable vector functions is always either convergent almost everywhere or divergent almost everywhere.

Theorems 32 and 33 together give, when translated into the language of the calculus of probability, a new solution of the convergence problem for series $x_{1}+x_{2}+\cdots$ whose terms are independent random variables; this problem (for $k=1$ ) was first treated by Khintchine and Kolmogoroff [37] and later by Kolmogoroff [38] and Lévy [41]. Theorem 33 states that the probability for convergence is always either 0 or 1 and Theorem 32 shows that the probability is 1 if and only if the distribution function $\psi_{n}$ of $s_{n}=x_{1}+\cdots+x_{n}$ tends to a distribution function $\psi$ when $n \rightarrow \infty$. Combining Theorem 5 with a remark due to Khintchine and Kolmogoroff, we find also the main result of these authors, which we formulate as a convergence criterion for infinite convolutions. Denoting by $C_{K}$ the sphere $|x| \leqq K$ and by $\phi_{n, K}$ the distribution function for which $\phi_{n, K}(E)$ is $=\phi_{n}\left(E C_{K}\right)+1-\phi_{n}\left(C_{K}\right)$ or $=\phi_{n}\left(E C_{K}\right)$ according as $E$ does or does not contain the point $x=0$, we have

THEOREM 34. A necessary and sufficient condition for the convergence of the infinite convolution $\phi_{1} * \phi_{2} * \cdots$ is the convergence of the three series

$$
\begin{gathered}
\left(1-\phi_{1}\left(C_{K}\right)\right)+\left(1-\phi_{2}\left(C_{K}\right)\right)+\cdots, \\
c\left(\phi_{1, K}\right)+c\left(\phi_{2, K}\right)+\cdots \text { and } \mathbf{M}_{2}\left(\tilde{\phi}_{1, K}\right)+\mathrm{M}_{2}\left(\tilde{\phi}_{2, K}\right)+\cdots
\end{gathered}
$$

for a fixed $K>0$ (or for all $K>0$ ). 
With the notation of Theorem 32, let $x_{n, K}\left(\tau_{n}\right)$ be $=x_{n}\left(\tau_{n}\right)$ when $\left|x_{n}\left(\tau_{n}\right)\right| \leqq K$ and $=0$ when $\left|x_{n}\left(\tau_{n}\right)\right|>K$ so that $x_{n, K}\left(\tau_{n}\right)$ has $\phi_{n, K}$ as distribution function. On combining Theorems 5 and 32 we see that the convergence of the series $c\left(\phi_{1, K}\right)+c\left(\phi_{2, K}\right)+\cdots$ and $\mathbf{M}_{2}\left(\tilde{\phi}_{1, K}\right)+\mathrm{M}_{2}\left(\tilde{\phi}_{2, K}\right)+\cdots$ is necessary and sufficient for the convergence almost everywhere of $x_{1, K}\left(\tau_{1}\right)$ $+x_{2, K}\left(\tau_{2}\right)+\cdots$. On the other hand, $x_{1}\left(\tau_{1}\right)+x_{2}\left(\tau_{2}\right)+\cdots$ converges almost everywhere if and only if $\left(1-\phi_{1}\left(C_{K}\right)\right)+\left(1-\phi_{2}\left(C_{K}\right)\right)+\cdots$ is convergent and $x_{1, K}\left(\tau_{1}\right)+x_{2, K}\left(\tau_{2}\right)+\cdots$ converges almost everywhere.

As an application of Theorem $B$ we finally prove

THEOREM 35. If $\psi=\phi_{1} * \phi_{2} * \cdots$ is a convergent infinite convolution of distribution functions $\phi_{n}$ each of which is purely discontinuous, then $\psi$ is either purely discontinuous or singular or absolutely continuous.

Using the notation of Theorem 32, we may suppose that each $x_{n}\left(\tau_{n}\right)$ takes on an at most enumerable set of values $x_{n, 1}, x_{n, 2}, \cdots$. Let $M$ denote the smallest modul in $R_{x}$ containing all points $x_{n, m}$ so that for an arbitrary set $E$ in $R_{x}$ the vectorial sum $E+M$ is at most enumerable if $E$ is at most enumerable and $E+M$ is a null-set if $E$ is a null-set. If $E$ is a Borel set then the set $A$ of those points $t$ in $Q$ for which $s(t)=x_{1}\left(\tau_{1}\right)+x_{2}\left(\tau_{2}\right)+\cdots$ is convergent and belongs to $E+M$ satisfies the conditions of Theorem $\mathrm{B}$, so that $m(A)=1$ whenever $m(A)>0$. This means that $\psi(E+M)=1$ whenever $\psi(E+M)>0$ and a fortiori when $\psi(E)>0$ which implies Theorem 35 .

Theorem 35 has been used already in $\$ 6$. Further infinite convolutions of the type considered in Theorem 35 have recently been investigated by Schoenberg $[47]$ in connection with distribution problems for arithmetical functions.

\section{BIBLIOGRAPHY}

[1] A. S. Besicovitch, Almost Periodic Functions. Cambridge, 1932.

[2] S. Bochner, Monotone Funktionen, Stieltjessche Integrale und harmonische Analyse. Mathematische Annalen, vol. 108 (1933), pp. 378-410.

[3] S. Bochner and B. Jessen, Distribution functions and positive definite functions. Annals of Mathematics, vol. 35 (1934), pp. 252-257.

[4] H. Bohr, Om de Värdier den Riemann'ske Funktion $\zeta(\sigma+i t)$ antager $i$ Halvplanen $\sigma>1$. Proceedings of the Second Congress of Scandinavian Mathematicians, Copenhagen, 1911, pp. 113121.

[5] - Über das Verhalten von $\zeta(s)$ in der Halbebene $\sigma>1$. Göttinger Nachrichten, 1911, pp. 409-428.

[6] Lösung des absoluten Konvergenzproblems einer allgemeinen Klasse Dirichletscher Reihen. Acta Mathematica, vol. 36 (1913), pp. 197-240.

[7] - Om Addition af uendelig mange konvekse Kurver. Danske Videnskabernes Selskab, Forhandlinger, 1913, pp. 325-366.

[8] Sur la fonction $\zeta(s)$ dans le demi-plan $\sigma>1$. Comptes Rendus, vol. 154 (1912), pp. 1078-1081.

[9] —Über die Funktion $\zeta^{\prime}(s) / \zeta(s)$. Journal für Mathematik, vol. 141 (1912), pp. 217-234. 
[10] - Über die Bedeutung der Potenzreihen unendlich vieler Variablen in der Theorie der Dirichletschen Reihen $\sum a_{n} / n^{s}$. Göttinger Nachrichten, 1913, pp. 441-488.

[11] - Sur la fonction $\zeta(s)$ de Riemann. Comptes Rendus, vol. 158 (1914), pp. 1986-1988.

[12] Zur Theorie der Riemannschen Zetafunktion im kritischen Streifen. Acta Mathematica, vol. 40 (1915), pp. 67-100.

[13] Zur Theorie der allgemeinen Dirichletschen Reihen. Mathematische Annalen, vol. 79 (1918), pp. 136-156.

[14] - Über diophantische Approximationen und ihre Anwendungen auf Dirichletsche Reihen, besonders auf die Riemannsche Zetafunktion. Proceedings of the Fifth Congress of Scandinavian Mathematicians, Helsingfors, 1922, pp. 131-154.

[15] - Om Addition af konvekse Kurver med givne Sandsynlighedsfordelinger. Matematisk Tidsskrift B, 1923, pp. 10-15.

[16] Zur Theorie der fastperiodischen Funktionen I. Acta Mathematica, vol. 45 (1924), pp. 29-127.

[17] - Kleinere Beiträge zur Theorie der fastperiodischen Funktionen II. Danske Videnskabernes Selskab, Mathematisk-Fysiske Meddelelser, vol. 10, No. 6 (1930), pp. 12-17.

[18] H. Bohr and R. Courant, Neue Anwendungen der Theorie der diophantischen Approximationen auf die Riemannsche Zetafunktion. Journal für Mathematik, vol. 144 (1914), pp. 249-274.

[19] H. Bohr and B. Jessen, Om Sandsynlighedsfordelinger ved Addition af konvekse Kurver. Danske Videnskabernes Selskab, Skrifter, (8), vol. 12, No. 3 (1929).

[20] — Über die Werteverteilung der Riemannschen Zetafunktion. Acta Mathematica, vol. 54 (1930), pp. 1-35, vol. 58 (1932), pp. 1-55.

[21] Mean-value theorems for the Riemann zeta-function. Quarterly Journal of Mathematics, vol. 5 (1934), pp. 43-47.

[22] T. Carleman, Sur les équations intégrales singulières à noyau reél et symétrique. Uppsala, 1923.

[23] J. Favard, Sur la répartition des points où une fonction presque-périodique prend une valeur donnée. Comptes Rendus, vol. 194 (1932), pp. 1714-1716.

[24] — Leçons sur les Fonctions Presque-Périodiques. Paris, 1933.

[25] M. Fréchet and J. Shohat, $A$ proof of the general second limit-theorem in the theory of probability. These Transactions, vol. 33 (1931), pp. 533-543.

[26] E. K. Haviland, On the addition of convex curves in Bohr's theory of Dirichlet series. American Journal of Mathematics, vol. 55 (1933), pp. 332-334.

[27] On statistical methods in the theory of almost periodic functions. Proceedings of the National Academy of Sciences, vol. 19 (1933), pp. 549-555.

[28] On distribution functions and their Laplace-Fourier transforms. Proceedings of the National Academy of Sciences, vol. 20 (1934), pp. 50-57.

[29] On the theory of absolutely additive distribution functions. American Journal of Mathematics, vol. 56 (1934), pp. 625-658.

[30] E. Hille and J. D. Tamarkin, Remarks on a known example of a monotone continuous function. American Mathematical Monthly, vol. 36 (1929), pp. 255-264.

[31] B. Jessen, Bidrag til Integralteorien for Funktioner af uendelig mange Variable. Copenhagen, 1930.

[32] — Eine Integrationstheorie für Funktionen unendlich vieler Veränderlichen, mit Anwendung auf das Werteverteilungsproblem für fastperiodische Funktionen, insbesondere für die Riemannsche Zetafunktion. Verhandlungen des Internationalen Mathematikerkongresses, Zürich, 1932, vol. 2, pp. 135-136, and Matematisk Tidsskrift B, 1932, pp. 59-65.

[33] _Über die Nullstellen einer analytischen fastperiodischen Funktion. Eine Verallgemeinerung der Jensenschen Formel. Mathematische Annalen, vol. 108 (1933), pp. 485-516.

[34] $\longrightarrow$ note on distribution functions. Journal of the London Mathematical Society, vol. 8 (1933), pp. 247-250.

[35] The theory of integration in a space of an infinite number of dimensions. Acta Mathematica, vol. 63 (1934), pp. 249-323. 
[36] Some analytical problems relating to probability, Journal of Mathematics and Physics, vol. 14 (1935), pp. 24-27.

[37] A. Khintchine and A. Kolmogoroff, Über Konvergenz von Reihen, deren Glieder durch den Zufall bestimmt werden. Recueil de la Société Mathématique de Moscou, vol. 32 (1925), pp. 668-677.

[38] A. Kolmogoroff, Über die Summen durch den Zufall bestimmter zufälliger Grössen. Mathematische Annalen, vol. 99 (1928), pp. 309-319, vol. 102 (1930), pp. 484-488.

[39] E. Landau, Vorlesungen über Zahlentheorie, vol. 2. Leipzig, 1927.

[40] P. Lévy, Calcul des Probabilités. Paris, 1925.

[41] - Sur les séries dont les termes sont des variables éventuelles indépendentes. Studia Mathematica, vol. 3 (1931), pp. 119-155.

[42] R. Lüneburg, Das Problem der Irrfahrt ohne Richtungsbeschränkung und die Randwertaufgabe der Potentialtheorie. Mathematische Annalen, vol. 104 (1931), pp. 700-738.

[43] D. Menchoff, Sur l'unicité du développement trigonométrique. Comptes Rendus, vol. 163 (1916), pp. 433-436.

[44] R. E. A. C. Paley and A. Zygmund, On some series of functions. Proceedings of the Cambridge Philosophical Society, vol. 26 (1930), pp. 337-357 and 458-474, vol. 28 (1932), pp. 190-205.

[45] J. Radon, Theorie und Anwendung der absolut additiven Mengenfunktionen. Wiener Sitzungsberichte, vol. 122 (1913), pp. 1295-1438.

[46] Lord Rayleigh, On the problem of random vibrations, and of random fights in one, two or three dimensions. Philosophical Magazine, (6), vol. 37 (1919), pp. 321-347.

[47] I. J. Schoenberg, On infinite convolutions and arithmetical functions. To appear in these Transactions.

[48] H. Weyl, Über die Gleichverteilung von Zahlen mod. Eins. Mathematische Annalen, vol. 77 (1916), pp. 313-352.

[49] A. Wintner, Über den Konvergenzbegriff der mathematischen Statistik. Mathematische Zeitschrift, vol. 28 (1928), pp. 476-480.

[50] Spektraltheorie der unendlichen Matrizen. Leipzig, 1929.

[51] Diophantische Approximationen und Hermitesche Matrizen. I. Mathematische Zeitschrift, vol. 30 (1929), pp. 290-319.

[52] On the asymptotic repartition of the values of real almost periodic functions. American Journal of Mathematics, vol. 54 (1932), pp. 339-345.

[53] - On an application of diophantine approximations to the repartition problems of dynamics. Journal of the London Mathematical Society, vol. 7 (1932), pp. 242-246.

[54] _Über die statistische Unabhängigkeit der asymptotischen Verteilungsfunktionen inkommensurabler Partialschwingungen. Mathematische Zeitschrift, vol. 36 (1933), pp. 618-629, vol. 37 (1933), pp. 479-480.

[55] Upon a statistical method in the theory of diophantine approximations. American Journal of Mathematics, vol. 55 (1933), pp. 309-331.

[56] $A$ note on the non-differentiable function of Weierstrass. American Journal of Mathematics, vol. 55 (1933), pp. 603-605.

[57] On the addition of independent distributions. American Journal of Mathematics, vol. 56 (1934), pp. 8-16.

[58] - On the asymptotic differential distribution of almost-periodic and related functions. American Journal of Mathematics, vol. 56 (1934), pp. 401-406.

[59] On analytic convolutions of Bernoulli distributions. American Journal of Mathematics, vol. 56 (1934), pp. 659-663.

[60] On symmetric Bernoulli convolutions. Bulletin of the American Mathematical Society, vol. 41 (1935), pp. 137-138.

Institute For Advanced Study,

Princeton, N.J.

JOHNS HOPKINS UNIVERSITY,

BALTIMORE, MD. 